\title{
State Heterogeneity Analysis of Financial Volatility Using High-Frequency Financial Data
}

\author{
Dohyun Chun ${ }^{1}$ and Donggyu Kim ${ }^{1 *}$ \\ ${ }^{1}$ College of Business, Korea Advanced Institute of Science and Technology (KAIST), Seoul, Korea
}

March 1, 2021

\begin{abstract}
Recently, to account for low-frequency market dynamics, several volatility models, employing high-frequency financial data, have been developed. However, in financial markets, we often observe that financial volatility processes depend on economic states, so they have a state heterogeneous structure. In this paper, to study state heterogeneous market dynamics based on high-frequency data, we introduce a novel volatility model based on a continuous Itô diffusion process whose intraday instantaneous volatility process evolves depending on the exogenous state variable, as well as its integrated volatility. We call it the state heterogeneous GARCH-Itô (SG-Itô) model. We suggest a quasi-likelihood estimation procedure with the realized volatility proxy and establish its asymptotic behaviors. Moreover, to test the low-frequency state heterogeneity, we develop a Wald test-type hypothesis testing procedure. The results of empirical studies suggest the existence of leverage, investor attention, market illiquidity, stock market comovement, and post-holiday effect in S\&P 500 index volatility.
\end{abstract}

\section{JEL classification: C22, C53, C58}

Key words and phrases: GARCH, diffusion process, regime switching, quasi-maximum likelihood estimator, Wald test.

\footnotetext{
* corresponding author.

E-mail addresses: dohyun0323@kaist.ac.kr (D. Chun), donggyukim@kaist.ac.kr (D. Kim).
} 


\section{Introduction}

Volatility plays an important role in financial asset pricing, risk management, portfolio allocation, and managerial decision-making. These interests have led many researchers to analyze financial volatility features such as time-varying heteroscedasticity, heavy tailness, and volatility clustering effect. To account for stylized market features, GARCH models (Bollerslev, 1986, Engle, 1982) have been introduced. In financial markets, we often observe that volatility varies with economic or financial states, but the plain GARCH model cannot deal with this. To consider this state heterogeneity in the volatility process, researchers have developed state-heterogeneity GARCHtype models - for example, Markov-switching GARCH (Bauwens et al., 2010, 2014, Gray, 1996 , Haas et al., 2004; Hamilton and Susmel, 1994; Klaassen, 2002), GJR-GARCH (Glosten et al. 1993), and QR-GARCH (Nyberg, 2012) models. Their empirical studies support the existence of state heterogeneity in financial volatility.

GARCH family models generally use daily return information to determine daily volatility levels, but daily return squares provide limited information about current volatility levels Andersen and Bollerslev, 1998). Therefore, the data period should be long enough to enjoy the large-sample asymptotic properties of estimator. However, structural breaks in long-time-period data may deteriorate the estimation quality and the requirement for long-time-period data hinders investigation of short-term market dynamics. State heterogeneity models are severely limited in their exposure to the aforementioned issues because the number of parameters increases in proportion to the number of states, and the data are split among states. Recently, widely available financial big data have shed light on this issue. For example, thanks to advances of technology, high-frequency financial data are available, and we can accurately estimate volatility with relatively short-timeperiod data. In particular, researchers have modeled high-frequency data based on continuous-time Itô processes and proposed procedures for estimating realized volatility. Examples include multiscale realized volatility (Zhang, 2006, 2011), pre-averaging realized volatility (Jacod et al., 2009), quasi-maximum likelihood estimator (QMLE; Ä̈t-Sahalia et al., 2010, Xiu, 2010), kernel realized volatility (Barndorff-Nielsen et al. 2008), and robust pre-averaging realized volatility (Fan and 
Kim, 2018). Renault and Werker (2011) suggested the endogenous trading time robust realized volatility, and Liu et al. (2018) demonstrated that the pre-averaging estimator is robust for the zero-duration high-frequency data. The availability of these efficient realized volatility estimators had made a great impact on the volatility modeling and analysis. For example, in regard to the modeling aspect, researchers have tried to bridge the gap between the discrete-time volatility model and continuous-time process (Kallsen and Taqqu, 1998; Nelson, 1990; Wang, 2002). For the volatility dynamics analysis, realized volatility is employed as an innovation, which helps to improve estimation and prediction performance (Cerovecki et al., 2019, Engle and Gallo, 2006. Kim and Wang, 2020; Shephard and Sheppard, 2010; Song et al., 2021; Tao et al., 2011; Visser, 2011). Recently, Kim and Wang (2016) introduced the unified continuous volatility process (unified GARCH-Itô model) to provide a mathematical background for using high-frequency data in the GARCH model estimation. They showed that incorporating high-frequency financial data improves parameter estimation performance and helps analyze low-frequency market dynamics. See also Kim (2016); Kim and Fan (2019). In this manner, some state heterogeneous volatility models also incorporate high-frequency data. For instance, researchers employed realized volatility in regime-switching ARMA-GARCH (Zhang and Frey, 2015), two-stage three-state FIGARCH (Shi and Ho, 2015), realized GARCH (Hansen et al., 2012), and multivariate Markov regime-switching GARCH (Lai et al., 2017) models. These studies reported the usefulness of high-frequency data in analyzing state heterogeneity in low-frequency financial volatility. The success of previous studies have increased interest in developing volatility models that provide a mathematical background for using high-frequency data to analyze low-frequency volatility dynamics.

To examine and account for state heterogeneity in low-frequency volatility dynamics based on high-frequency financial data, we propose a novel volatility model based on a continuous-time Itô diffusion process whose instantaneous volatility process evolves depending on the state variables. In particular, its instantaneous volatility process is continuous with respect to time and has a homogeneous process during each low-frequency period. In contrast, the process varies with the state at each low-frequency period to capture the low-frequency market dynamics. Consequently, its 
integrated volatility process has a form of the famous regime switching GARCH model. The model is called the state heterogeneous GARCH-Itô (SG-Itô) model. To estimate model parameters, we suggest a quasi-maximum likelihood estimation procedure based on the high-frequency data and establish its asymptotic theories. Furthermore, to test state heterogeneity in low-frequency volatility, we introduce a Wald test-type hypothesis testing procedure. The results of empirical studies suggest the existence of leverage, trading volume or investor attention, market illiquidity, stock market comovement, and post-holiday effect on S\&P 500 index volatility. However, these state heterogeneities are not revealed with the same period of low-frequency data, because of its inefficiency. More details are provided in Section 5.2 .

The rest of the paper is organized as follows. Section 2 introduces the SG-Itô model and illustrates properties of instantaneous and integrated volatility processes. Section 3 presents the quasi-maximum likelihood method and establishes its asymptotic theories. Section 4 suggests a hypothesis testing procedure. Section 5 provides the results of numerical studies, including simulation and empirical studies. Section 6 concludes the paper. The proofs are provided in the Appendix.

\section{State heterogeneous GARCH-Itô (SG-Itô) model}

\subsection{State heterogeneity in discrete-time volatility processes}

State heterogeneity in financial volatility has long been discussed as the key feature of market dynamics (Lamoureux and Lastrapes, 1990b). To account for state heterogeneity in the volatility process, researchers have proposed various form of regime-switching GARCH (RS-GARCH) models. For example, Hamilton and Susmel (1994) applied the Markov-switching approach to build the state heterogeneous GARCH process. See also Bauwens et al. (2010, 2014), Gray (1996), Haas et al. (2004), and Klaassen (2002). Glosten et al. (1993) introduced the GJR-GARCH model, which reflects the well-known leverage effect (Black, 1976; Christie, 1982, Figlewski and Wang, 2000, Tauchen et al., 1996). Taking the state of the business cycle into account exogenously, Nyberg 
(2012) introduced the regime-switching GARCH-M model (QR-GARCH) model to examine the state-dependent risk-return relationship.

A regime-switching model is characterized by the joint process of historical log price $\left\{X_{n}\right\}$ and state variable $\left\{s_{n}\right\}$. Specifically, a process of the state variable $s_{t}$ and an evolution process of $X_{t}$ for a given state identify the model structure (Lange and Rahbek, 2009). In the case of the RSGARCH model, the conditional volatility process depends on the sigma field generated by $\left\{X_{n}\right\}$ $\left(\mathcal{F}_{n}^{x, L}=\sigma\left(X_{n}, X_{n-1}, X_{n-2}, \ldots\right)\right)$ and $\left\{s_{n}\right\}\left(\mathcal{F}_{n}^{s}=\sigma\left(s_{n}, s_{n-1}, s_{n-2}, \ldots\right)\right)$, where $n \in \mathbb{N}$ and $\mathbb{N}$ is the set of all non-negative integers. A general discrete-time RS-GARCH model is described as follows:

$$
\begin{aligned}
& X_{n}-X_{n-1}=\mu+\sqrt{h_{n}\left(\theta^{s, L}\right)} \epsilon_{n}^{L}, \\
& h_{n}\left(\theta^{s, L}\right)=\omega_{i}^{L}+\gamma_{i}^{L} h_{n-1}\left(\theta^{s, L}\right)+\beta_{i}^{L} \zeta_{n-1}^{2},
\end{aligned}
$$

where $\theta^{s, L}=\left(\omega_{i}^{L}, \gamma_{i}^{L}, \beta_{i}^{L}\right)$ is a model parameter for the state indicator $i=1,2, \ldots, D, D$ is the number of states, $\mu$ is a drift, $\zeta_{n}=X_{n}-X_{n-1}-\mu$, and random error $\epsilon_{n}^{L}$ satisfies $E\left[\epsilon_{n}^{L} \mid \mathcal{F}_{n-1}^{L}\right]=0$

and $E\left[\left(\epsilon_{n}^{L}\right)^{2} \mid \mathcal{F}_{n-1}^{L}\right]=1$ a.s. for $\mathcal{F}_{n-1}^{L}=\mathcal{F}_{n-1}^{x, L} \cup \mathcal{F}_{n}^{s}$. For the RS-GARCH model, the model parameters vary with the state, so the state variable $s_{t}$ plays a key role. The state variable $s_{t}$ may have variety of forms, and the assumption for the state process distinguishes the model. For example, the Markov-switching GARCH model has a latent Markov state process, whereas the GJR- and QR-GARCH models employ exogenous state variables.

\subsection{State heterogeneous GARCH-Itô model}

In the study of discrete-time market dynamics, a long time period of data is needed to obtain consistent estimation results. However, the long period of data is prone to exposure to the structural break issue, especially for RS-GARCH-type models, because of their complexity. Recently, realized volatility estimators based on high-frequency financial data have been well developed (Aït-Sahalia et al., 2010; Barndorff-Nielsen et al., 2008; Jacod et al., 2009; Xiu, 2010; Zhang, 2006). Kim and Wang (2016) showed improvement of parameter estimation efficiency by using realized volatility estimators as the estimation proxy. Therefore, we hypothesize that (1) the state heterogeneity 
exists in financial volatility process and (2) using high-frequency data facilitates its analysis. For hypothesis testing, a model that enables to utilize realized volatility estimators in the analysis of low-frequency state heterogeneity is required. This section introduces a novel continuous-time volatility model whose instantaneous volatility process varies with a discrete state process.

For the state variable process $s_{n}$, we consider discrete-time exogenous variables that determine the state of volatility process. The term exogenous comes from the independence assumption between the state variable $s_{n}$ and price process, which describe the unilateral influence of the state on the volatility process. For simplicity, this study deals with the binary state by assuming $\left\{s_{n}\right\}$ as a binary process. Let $\mathbb{R}^{+}=[0, \infty]$ and $t \in \mathbb{R}^{+}$. We define a state heterogeneity volatility model with a continuous-time Itô process as follows.

Definition 1. For $t \in(n-1, n]$, we call a log stock price $X_{t}$ follows an SG-Itô model if it satisfies

$$
\begin{aligned}
& d X_{t}=\mu d t+\sigma_{t} d B_{t}, \quad \sigma_{t}=\left(1-s_{n}\right) \sigma_{1, t}+s_{n} \sigma_{2, t}, \\
& \sigma_{i, t}^{2}=\sigma_{n-1}^{2}+(t-n+1)\left\{\omega_{i}+\left(\gamma_{i}-1\right) \sigma_{n-1}^{2}\right\}+\beta_{i}\left(\int_{n-1}^{t} \sigma_{i, s} d B_{s}\right)^{2} \text { for } i \in\{1,2\},
\end{aligned}
$$

where $X_{t}$ is a log stock price, $\sigma_{1, t}^{2}$ and $\sigma_{2, t}^{2}$ are volatility processes adapted to $\mathcal{F}_{t}^{x}=\sigma\left(X_{s}: s \leq t\right)$, $B_{t}$ is the standard Brownian motion with respect to a filtration $\mathcal{F}_{t}^{x}$, and $\theta=\left(\omega_{1}, \omega_{2}, \gamma_{1}, \gamma_{2}, \beta_{1}, \beta_{2}\right)$ are model parameters.

For the SG-Itô model, instantaneous volatility has a continuous-time state heterogeneous process defined at all times $t$. In particular, during the current low-frequency period $t \in(n-1, n]$, the instantaneous volatility $\sigma_{s_{n}+1, t}^{2}$ has a homogeneous continuous-time Itô process depending on the current state variable $s_{n}$. During the next low-frequency period $t \in(n, n+1]$, the instantaneous volatility $\sigma_{s_{n+1}+1, t}^{2}$ evolves from the end of the previous instantaneous process $\sigma_{s_{n}+1, n}^{2}$ while their evolving process is determined by the next period state variable $s_{n+1}$. This model deals with the low-frequency state heterogeneity in volatility by state-varying coefficients. For example, for $s_{n}=0$, we have $\left(\omega_{i}, \gamma_{i}, \beta_{i}\right)=\left(\omega_{1}, \gamma_{1}, \beta_{1}\right)$ and $\sigma_{t}^{2}=\sigma_{1, t}^{2}$, whereas for $s_{n}=1$, we have $\left(\omega_{i}, \gamma_{i}, \beta_{i}\right)=\left(\omega_{2}, \gamma_{2}, \beta_{2}\right)$ and $\sigma_{t}^{2}=\sigma_{2, t}^{2}$. Moreover, the current level of volatility depends on past volatility due to its recursive 
structure. Thus, the model is uniquely identified by the path of the state variables because the sequential order of the states differentiates the volatility process. Accordingly, it can handle the regime shift in volatility with the corresponding state variable. Since the model can incorporate any state process, it allows us to test a given exogenous state. This is a distinguishing feature of the SG-Itô model compared to the single-regime model. When the states are homogeneous $\left(s_{n}=s_{n-1}=\cdots=s_{1}\right)$, the model returns to a single-regime model, the unified GARCH-Itô model Kim and Wang, 2016). That is, the unified GARCH-Itô model is a special example of the SG-Itô model. More details are provided in Appendix A.2.

In this paper, we consider the case where $n \in \mathbb{N}$ denotes a day. In this case, $s_{n}$ is a daily state variable, and $\sigma_{t}^{2}$ for $t \in[n-1, n)$ denotes the intraday volatility on day $n$. Definition 1 now implies that intraday volatility on day $n$ evolves from close volatility on day $n-1$ reflecting the contemporaneous log stock price, while the evolving process depends on the daily state variable $s_{n}$.

\subsection{Integrated volatility for the SG-Itô model}

This study aims to investigate the low-frequency market dynamics, so the integrated volatility structure over the low-frequency period is important. Moreover, the integrated volatility process will be used in the parameter estimation procedure. In this section, we study properties of the integrated volatilities.

Theorem 1. (a) Under the SG-Itô framework, integrated volatility on state $i \in\{1,2\}$ can be decomposed into $\left\{\mathcal{F}_{n-1}^{x, L}, \mathcal{F}_{n-1}^{s}\right\}$-adapted process and martingale difference as follows:

$$
\int_{n-1}^{n} \sigma_{i, t}^{2} d t=h_{i, n}(\theta)+\xi_{i, n} \text { a.s. }
$$

where

$$
\begin{aligned}
& h_{i, n}(\theta)=H_{c, i}(\theta)+H_{\beta, i}(\theta) \sigma_{n-1}^{2}, \quad \xi_{i, n}=2 \int_{n-1}^{n}\left(e^{(n-t) \beta_{i}}-1\right) \int_{n-1}^{t} \sigma_{i, s} d B_{s} \sigma_{i, t} d B_{t}, \\
& H_{c, i}(\theta)=\beta_{i}^{-2}\left(e^{\beta_{i}}-1-\beta_{i}\right) \omega_{i}, H_{\beta, i}(\theta)=\left(\gamma_{i}-1\right) \beta_{i}^{-2}\left(e^{\beta_{i}}-1-\beta_{i}\right)+\beta_{i}^{-1}\left(e^{\beta_{i}}-1\right) .
\end{aligned}
$$


(b) Let $\mathcal{F}_{n-1}=\mathcal{F}_{n-1}^{x} \cup \mathcal{F}_{n}^{s}$. Then, for given $s_{n}$ and $s_{n-1}$, the conditional expected integrated volatility $E\left[\int_{n-1}^{n} \sigma_{t}^{2} d t \mid \mathcal{F}_{n-1}\right]=h_{n}(\theta)$ a.s. is represented by

$$
\begin{aligned}
h_{n}(\theta)= & s_{11, n}\left(\omega_{11}^{h}+\gamma_{11}^{h} h_{n-1}(\theta)+\beta_{11}^{h} Z_{n-1}^{2}\right)+s_{12, n}\left(\omega_{12}^{h}+\gamma_{12}^{h} h_{n-1}(\theta)+\beta_{12}^{h} Z_{n-1}^{2}\right) \\
& +s_{21, n}\left(\omega_{21}^{h}+\gamma_{21}^{h} h_{n-1}(\theta)+\beta_{21}^{h} Z_{n-1}^{2}\right)+s_{22, n}\left(\omega_{22}^{h}+\gamma_{22}^{h} h_{n-1}(\theta)+\beta_{22}^{h} Z_{n-1}^{2}\right),
\end{aligned}
$$

where

$$
\begin{aligned}
& s_{11, n}=\left(1-s_{n-1}\right)\left(1-s_{n}\right), s_{12, n}=\left(1-s_{n-1}\right) s_{n}, s_{21, n}=s_{n-1}\left(1-s_{n}\right), s_{22, n}=s_{n-1} s_{n}, \\
& \theta^{h}=\left\{\omega_{11}^{h}, \omega_{12}^{h}, \omega_{21}^{h}, \omega_{22}^{h}, \gamma_{11}^{h}, \gamma_{12}^{h}, \gamma_{21}^{h}, \gamma_{22}^{h}, \beta_{11}^{h}, \beta_{12}^{h}, \beta_{21}^{h}, \beta_{22}^{h}\right\}, \\
& \omega_{i i}^{h}=\left(1-\gamma_{i}\right) H_{c, i}(\theta)+\omega_{i} H_{\beta, i}(\theta), \gamma_{i i}^{h}=\gamma_{i}, \beta_{i i}^{h}=\beta_{i} H_{\beta, i}(\theta), \\
& \omega_{i j}^{h}=H_{c, j}(\theta)-\gamma_{i} H_{c, i}(\theta)\left(H_{\beta, j}(\theta) / H_{\beta, i}(\theta)\right)+\omega_{i} H_{\beta, j}(\theta), \gamma_{i j}^{h}=\gamma_{i}\left(H_{\beta, j}(\theta) / H_{\beta, i}(\theta)\right), \beta_{i j}^{h}=\beta_{i} H_{\beta, j}(\theta), \\
& Z_{n}=\left(1-s_{n}\right) Z_{1, n}+s_{n} Z_{2, n}, Z_{i, t}=\int_{t-1}^{t} \sigma_{i, s} d B_{s} \text { for } i \in\{1,2\} .
\end{aligned}
$$

Theorem 1(a) shows that integrated volatility on state $i$ is decomposed into GARCH volatility $h_{i, n}(\theta)$ and martingale difference $\xi_{i, n}$. This decomposition plays a prominent role in the subsequent theorems, and we show that the theorems can be established for any process that satisfies the decomposition in Theorem 1(a). Theorem 1(b) indicates that the expected integrated volatility $h_{n}(\theta)$ follows a four-state RS-GARCH$(1,1)$ structure. In particular, the integrated form of the model parameter $\theta^{h}$ is determined by the product of $s_{n}$ and $s_{n-1}$, so the integrated volatility depends on both current and previous states. In the sense that its integrated volatility has a RSGARCH-like structure, the SG-Itô model has an instantaneous volatility process that characterizes the RS-GARCH models. We note that this general model allows to incorporate and extend existing regime-switching volatility frameworks by employing a suitable state process. For example, this model illustrates the Markov-switching GARCH model with latent Markov state process and the GJR- and QR-GARCH models with observed exogenous state processes.

In this paper, we mainly deal with observed state processes. In practice, however, future state is often unobservable. For instance, day-of-week or previous day return state is available at the 
beginning of the day, whereas daily trading volume or market illiquidity is not available until the end of the day. We note that the SG-Itô model does not require the observability of the state variable $s_{n}$. For unrevealed $s_{n}$, Proposition 1 suggests that we can estimate the expected integrated volatility with state transition probability.

Proposition 1. For unrevealed $s_{n}$ and given $s_{n-1}$, we have

$$
\begin{aligned}
E\left[\int_{n-1}^{n} \sigma_{t}^{2} d t \mid \mathcal{F}_{n-1}^{x}, \mathcal{F}_{n-1}^{s}\right]= & p_{11, n}\left(1-s_{n-1}\right)\left(\omega_{11}^{h}+\gamma_{11}^{h} h_{n-1}(\theta)+\beta_{11}^{h} Z_{n-1}^{2}\right) \\
& +p_{12, n}\left(1-s_{n-1}\right)\left(\omega_{12}^{h}+\gamma_{12}^{h} h_{n-1}(\theta)+\beta_{12}^{h} Z_{n-1}^{2}\right) \\
& +p_{21, n} s_{n-1}\left(\omega_{21}^{h}+\gamma_{21}^{h} h_{n-1}(\theta)+\beta_{21}^{h} Z_{n-1}^{2}\right) \\
& +p_{22, n} s_{n-1}\left(\omega_{22}^{h}+\gamma_{22}^{h} h_{n-1}(\theta)+\beta_{22}^{h} Z_{n-1}^{2}\right) \text { a.s. },
\end{aligned}
$$

where $p_{i j, n}=p\left(s_{n}=j-1 \mid s_{n-1}=i-1\right)$ for $i, j \in\{1,2\}$.

In practice, $Z_{n}$ 's are not observable due to the drift term $\mu$, thus to predict the future volatility, we first need to estimate $\mu$ using the sample mean of the daily log-returns. The martingale convergence theorem shows that the sample mean of daily log-return converges to $\mu$.

\section{Estimation procedure}

\subsection{Model setup}

In this paper, we assume that the true log price process follows the SG-Itô model as described in Definition 1. We also distinguish the low- and high-frequency data as follows. The low-frequency data signify the log price observed at integer time points $t=0,1,2, \ldots$ and we assume that the true low-frequency log prices, $X_{0}, X_{1}, \ldots$, are observed. At the same time, high-frequency data indicate the log price observed at time points between integer time points, which are denoted by $t_{n, m}$ for $n=0,1, \ldots, N$ and $m=1, \ldots, M_{n}-1$ for each $n$ and satisfy $n-1=t_{n, 0}<t_{n, 1}<\cdots<$ $t_{n, M_{n}}=t_{n+1,0}=n$. In the high-frequency finance, the observed price $Y_{t_{n, m}}$ is contaminated by micro-structure noises. To reflect this, we assume that $Y_{t_{n, m}}$ is composed of the true price $X_{t_{n, m}}$ 
and micro-structure noise $\epsilon_{t_{n, m}}$ as follows:

$$
Y_{t_{n, m}}=X_{t_{n, m}}+\epsilon_{t_{n, m}}
$$

where $\epsilon_{t_{n, m}}$ is independent with the price and volatility process and i.i.d. with mean zero and standard deviation $\sigma_{\epsilon}$. For the low-frequency data, we can estimate drift easily by calculating the sample mean of the low-frequency data. For the high-frequency based realized volatility estimator, the effect of drift is asymptotically negligible. Therefore, for simplicity, we assume $\mu=0$ in Equations (2.1) and (2.2). This is not a necessary condition but allows us to focus more on developing and analyzing volatility processes.

\subsection{Quasi-maximum likelihood estimation}

For convenience, we first review some notations and definitions. Unless stated otherwise, limits are taken as $N, M \rightarrow \infty$, where $M=\sum_{n=1}^{N} M_{n} / N$. Let $\stackrel{p}{\longrightarrow}$ and $\stackrel{d}{\longrightarrow}$ be convergence in probability and distribution, respectively. The $L_{p}$ norm of a random variable $\mathrm{Z}$ is denoted by $\|Z\|_{L_{p}}=\left(E\left[|Z|^{p}\right]\right)^{\frac{1}{p}}$. Finally, $\|X\|_{\max }=\max _{j, k}\left|X_{j, k}\right|$ for a matrix $X=\left(X_{j, k}\right)_{j, k=1, \ldots, q}$ and $\|x\|_{\max }=\max _{j}\left|x_{j}\right|$ for a vector $x=\left(x_{1}, \ldots, x_{q}\right)$. C's present a positive generic constant whose values can be changed from appearance to appearance, free from $\theta, N$, and $M_{n}$.

For statistical inferences, we apply a quasi-maximum likelihood estimation procedure to the integrated volatility process. Theorem 1(a) suggests that integrated volatility over the $n$th period is decomposed into the GARCH volatility $h_{n}(\theta)$ and martingale difference. The well-developed martingale convergence theorem indicates that the integrated volatility converges to $h_{n}\left(\theta_{0}\right)$ as $N \rightarrow$ $\infty$, so the integrated volatility can be a good proxy of $h_{n}\left(\theta_{0}\right)$. Unfortunately, integrated volatility is not observed, so we need to estimate it using the observed noisy high-frequency data. There are well-performing realized volatility estimators such as multi-scale realized volatility (Zhang, 2006 , 2011), pre-averaging realized volatility (Jacod et al., 2009), and kernel realized volatility (BarndorffNielsen et al., 2008), which have the optimal convergence rate $M_{n}^{-1 / 4}$. We adopt the pre-averaging realized volatility in the numerical studies. 
Let $\theta=\left(\omega_{1}, \omega_{2}, \beta_{1}, \beta_{2}, \gamma_{1}, \gamma_{2}\right) \in \Theta$ with the true value $\theta_{0}=\left(\omega_{0,1}, \omega_{0,2}, \beta_{0,1}, \beta_{0,2}, \gamma_{0,1}, \gamma_{0,2}\right) \in \Theta$ for the compact parameter space $\Theta$. Then, for the given state variable $s_{n}$, the quasi-maximum likelihood function is defined as follows:

$$
\widehat{Q}_{N, M}(\theta)=-\frac{1}{2 N} \sum_{n=1}^{N}\left[\log \left(h_{n}(\theta)\right)+\frac{R V_{n}}{h_{n}(\theta)}\right],
$$

where $R V_{n}$ is the realized volatility estimator constructed based on high-frequency data during the $n$th period and $h_{n}(\theta)=\left(1-s_{n}\right) h_{1, n}(\theta)+s_{n} h_{2, n}(\theta)$ is state-heterogeneous GARCH volatility presented in Theorem 1(b). The estimation procedure can be easily generalized to the other forms of state processes with a suitable quasi-likelihood function. The QMLE $\widehat{\theta}$ is

$$
\widehat{\theta}=\underset{\theta \in \Theta}{\operatorname{argmax}} \widehat{Q}_{N, M}(\theta)
$$

To investigate the asymptotic behaviors of QMLE $\widehat{\theta}$, we need the following technical conditions.

\section{Assumption 1.}

(a) Let the parameter space $\Theta=\left\{\theta=\left(\omega_{1}, \omega_{2}, \gamma_{1}, \gamma_{2}, \beta_{1}, \beta_{2}\right): \omega_{l}<\omega_{i}<\omega_{u}, \gamma_{l}<\gamma_{i}<\gamma_{u}, \beta_{l}<\right.$ $\left.\beta_{i}<\beta_{u}, \omega_{l}^{h}<\omega_{i j}^{h}<\omega_{u}^{h}, \gamma_{l}^{h}<\gamma_{i j}^{h}<\gamma_{u}^{h}, \beta_{l}^{h}<\beta_{i j}^{h}<\beta_{u}^{h}, \gamma_{i j}^{h}+\beta_{i j}^{h}<1\right\}$ for $i, j \in\{1,2\}$, where $\omega_{l}, \omega_{u}, \gamma_{l}, \gamma_{u}, \beta_{l}, \beta_{u}, \omega_{l}^{h}, \omega_{u}^{h}, \gamma_{l}^{h}, \gamma_{u}^{h}, \beta_{l}^{h}, \beta_{u}^{h}$ are known positive constants.

(b) $\sup _{n \in \mathbb{N}} E\left(\left|\xi_{i, n}\right|^{1+\delta}\right)<\infty$ for $i \in\{1,2\}$ and some $\delta>0$.

(c) $\frac{E\left[Z_{n}^{4} \mid \mathcal{F}_{n-1}\right]}{h_{n}\left(\theta_{0}\right)^{2}} \leq C$ a.s. for any $n \in \mathbb{N}$.

(d) $\left\{\xi_{i, n}, Z_{i, n}, s_{n}\right\}$ is a stationary and ergodic process.

Remark 1. Assumption 1 is required to handle the low-frequency part. Assumption 1 (b) is the sufficient condition for the uniform integrability of martingale difference process. The uniform integrability is a necessary condition to show the boundedness of derivatives of the quasi-likelihood functions, which is required to obtain the consistency of $\widehat{\theta}$. Assumption 1 (c) is the finite fourth moment condition. Because the target parameter is the second moment, the finite fourth moment condition is not strong at all to obtain the convergence rate $N^{-1 / 2}$ (see also Lee and Hansen (1994)). 
Assumption 1 1 (d) is only required to derive asymptotic normality of $\widehat{\theta}$. However, this condition is not an obvious result under the SG-Itô model. It is an interesting theoretical problem to investigate conditions which imply Assumption 11(d) under the SG-Itô model. We leave this for the future study.

Remark 2. The state process should be stationary and ergodic. It includes state processes that are generally applied in existing switching models. For example, any multinomial variables of ergodic probabilities are included. Specifically, the state variable $s_{t}$ satisfies

$$
s_{t} \mid \Omega_{t-1} \sim \operatorname{Bernoulli}\left(p_{t}\right), \quad p_{t}=E_{t-1}\left(s_{t}=1\right)=\Phi\left(\pi_{t}\right)
$$

where $\Phi(\cdot)$ is a link function and $\pi_{t}$ is explanatory variables. Then, under some stationary condition for $\pi_{t}$, the state variable $s_{t}$ is a stationary and ergodic process.

\section{Assumption 2.}

(a) Assume $C_{1} M \leq M_{n} \leq C_{2} M$, $\sup _{n} \sup _{1 \leq m \leq M_{n}}\left|t_{n, m}-t_{n, m-1}\right|=O\left(M^{-1}\right)$, and $N^{2} M^{-1} \rightarrow 0$ as $N, M \rightarrow \infty$.

(b) $\sup _{n \in \mathbb{N}}\left\|R V_{n}-\int_{n-1}^{n} \sigma_{t}^{2} d t\right\|_{L_{2}} \leq C M^{-\frac{1}{4}}$.

(c) For any $n \in \mathbb{N}, E\left[R V_{n} \mid \mathcal{F}_{n-1}\right] \leq C E\left[\int_{n-1}^{n} \sigma_{t}^{2} d t \mid \mathcal{F}_{n-1}\right]+C$ a.s.

Remark 3. Assumption 2 stands for the high-frequency part. Assumption 2(a) is a typical condition for realized volatility estimators. Assumption $2(b)-(c)$ can be obtained easily under some fourth moment conditions as discussed in Tao et al. (2013), Kim et al. (2016), and Kim et al. (2018).

Theorems 2 and 3 establish the consistency of $\widehat{\theta}$ and its convergence rate, respectively.

Theorem 2. Under Assumption $1(a)-(b)$ and Assumption $2(a)-(b)$, we have $\widehat{\theta} \rightarrow \theta_{0}$ in probability.

Theorem 3. Under Assumption 1 $1(a)-(c)$ and Assumption [2, we have $\left\|\hat{\theta}-\theta_{0}\right\|_{\max }=O_{p}\left(N^{-\frac{1}{2}}+\right.$ $\left.M^{-\frac{1}{4}}\right)$. 
Remark 4. Theorem 3 shows that the convergence rate of $\widehat{\theta}$ has both high- and low-frequencyoriented components. The rate $N^{-\frac{1}{2}}$ is due to the low-frequency part, which is the usual parametric convergence rate. The rate $M^{-\frac{1}{4}}$ is from the high-frequency volatility estimation related to Assumption 2(b), known as the optimal convergence rate of the realized volatility estimator with the presence of the micro-structure noise.

Theorem 4 derives asymptotic normality of $\widehat{\theta}$ using stationary and ergodic assumptions.

Theorem 4. Suppose that Assumptions 1 and 2 are met and

$$
\begin{aligned}
& \frac{1}{4 N} \sum_{n=1}^{N}\left[\left.\frac{\partial h_{n}(\theta)}{\partial \theta}\left[\frac{\partial h_{n}(\theta)}{\partial \theta}\right]^{T}\right|_{\theta=\theta_{0}} h_{n}\left(\theta_{0}\right)^{-4} \xi_{n}^{2}\right] \stackrel{p}{\longrightarrow} V, \\
& \frac{1}{2 N} \sum_{n=1}^{N}\left[\left.\frac{\partial h_{n}(\theta)}{\partial \theta}\left[\frac{\partial h_{n}(\theta)}{\partial \theta}\right]^{T}\right|_{\theta=\theta_{0}} h_{n}\left(\theta_{0}\right)^{-2}\right] \stackrel{p}{\longrightarrow} W .
\end{aligned}
$$

Then we have

$$
\sqrt{N}\left(\widehat{\theta}-\theta_{0}\right) \stackrel{d}{\longrightarrow} N\left(0, W^{-1} V W^{-1}\right) .
$$

Theorem 4 demonstrates that the limiting distribution of QMLE is Gaussian with the variance $W^{-1} V W^{-1}$, where the matrices $V$ and $W$ are information and Hessian matrices, respectively. Theorem 4 implies that the quality of the integrated volatility estimator affects the variance of the parameter estimates. To check the effect of employing integrated volatility estimators as the proxy, we consider the parameter estimation procedure using low-frequency data only. For example, the QMLE $\widehat{\theta}^{L}$ is obtained as follows:

$$
\widehat{Q}_{N}^{L}(\theta)=-\frac{1}{2 N} \sum_{n=1}^{N}\left[\log \left(h_{n}(\theta)\right)+\frac{\zeta_{n}^{2}}{h_{n}(\theta)}\right] \quad \text { and } \quad \widehat{\theta}^{L}=\underset{\theta \in \Theta}{\operatorname{argmax}} \widehat{Q}_{N}^{L}(\theta),
$$

where $\zeta_{n}=X_{n}-X_{n-1}-\mu$ is defined in Equation (2.1). Then, similar to the proof of Theorem 4 . 
the asymptotic distribution of $\widehat{\theta}^{L}$ can be derived as follows:

$$
\sqrt{N}\left(\widehat{\theta}^{L}-\theta_{0}\right) \stackrel{d}{\longrightarrow} N\left(0, W^{-1} V^{L} W^{-1}\right)
$$

where

$$
\frac{1}{4 N} \sum_{n=1}^{N}\left[\left.\frac{\partial h_{n}(\theta)}{\partial \theta}\left[\frac{\partial h_{n}(\theta)}{\partial \theta}\right]^{T}\right|_{\theta=\theta_{0}} h_{n}\left(\theta_{0}\right)^{-4}\left(Z_{n}^{2}-h_{n}\left(\theta_{0}\right)\right)^{2}\right] \stackrel{p}{\longrightarrow} V^{L} .
$$

From the above results, we can find that the estimation errors of the GARCH volatility in realized volatility (i.e., $\left.\int_{n-1}^{n} \sigma_{t}^{2} d t-h_{n}\left(\theta_{0}\right)\right)$ or daily return square (i.e., $\left.Z^{2}-h_{n}\left(\theta_{0}\right)\right)$ play a key role in the variance of parameter estimates. We can easily find that the estimation errors in realized volatility is smaller than that in the daily return square. That is, compared to the daily return square, the sufficient information in realized volatility estimators reduces the parameter estimation error and produces accurate parameter estimates with a relatively short time period of data (see also Kim and Wang $(2016))$.

To make inferences based on the asymptotic distribution derived in Theorem 4, we construct consistent estimators for $V$ and $W$ as follows:

$$
\begin{aligned}
& \widehat{V}=\frac{1}{4 N} \sum_{n=1}^{N}\left[\left.\frac{\partial h_{n}(\theta)}{\partial \theta}\left[\frac{\partial h_{n}(\theta)}{\partial \theta}\right]^{T}\right|_{\theta=\widehat{\theta}} h_{n}(\widehat{\theta})^{-4}\left(R V_{n}-h_{n}(\widehat{\theta})\right)^{2}\right], \\
& \widehat{W}=\frac{1}{2 N} \sum_{n=1}^{N}\left[\left.\frac{\partial h_{n}(\theta)}{\partial \theta}\left[\frac{\partial h_{n}(\theta)}{\partial \theta}\right]^{T}\right|_{\theta=\widehat{\theta}} h_{n}(\widehat{\theta})^{-2}\right] .
\end{aligned}
$$

To show the consistency of estimators, we make following additional assumptions.

\section{Assumption 3.}

(a) $\frac{E\left[Z_{n}^{8} \mid \mathcal{F}_{n-1}\right]}{h_{n}\left(\theta_{0}\right)^{4}} \leq C$ a.s. for any $n \in \mathbb{N}$.

(b) $\sup _{n \in \mathbb{N}}\left\|R V_{n}-\int_{n-1}^{n} \sigma_{t}^{2} d t\right\|_{L_{4}} \leq C M^{-\frac{1}{4}}$.

Remark 5. Assumption 3 is the finite eighth moment condition. The parameters, $V$ and $W$, of interest are functions of fourth moments. Thus, to establish their asymptotic theorems, we need the 
finite eighth moment condition.

Proposition 2. Under Assumptions 1 3, we have $\widehat{V} \rightarrow V$ and $\widehat{W} \rightarrow W$ in probability.

Remark 6. Proposition 2 shows the consistency of $\widehat{V}$ and $\widehat{W}$. We can obtain their convergence rates by imposing some additional condition. For example, by assuming that $\left\{h_{n}\left(\theta_{0}\right), \xi_{n}^{2}\right\}$ is a strong mixing sequence, Theorem 1.2 (Merlevede and Peligrad, 2000) shows that $\widehat{V}$ and $\widehat{W}$ have the convergence rate $N^{-1 / 2}+M^{-1 / 4}$.

\section{Testing state heterogeneity}

The main purpose of this paper is to investigate state heterogeneity in the volatility process. In the previous section, we propose a state-heterogeneous diffusion process that can incorporate the low-frequency state process. Under the SG-Itô model, state heterogeneity in the volatility process is illustrated by the state-varying parameters $\omega_{i}, \gamma_{i}, \beta_{i}$. Therefore, we can test the state heterogeneity by conducting a hypothesis test under the null hypothesis statement

$$
H_{0}: \omega_{1}=\omega_{2}, \gamma_{1}=\gamma_{2}, \beta_{1}=\beta_{2}
$$

In this section, we construct a Wald test-type hypothesis testing procedure with the null hypothesis $H_{0}$ for the QMLE. The rejection of the null hypothesis signifies that the external state distinguishes the model specification, which implies the existence of state heterogeneity in the volatility process. Let $R$ be the $v \times u$ restriction matrix with full row rank. Theorem 5 defines the Wald-type statistic and establishes its limiting distribution.

Theorem 5. Under Assumptions 13 and the null hypothesis of $R \theta_{0}=r$, we have

$$
T_{N, M}=N(R \widehat{\theta}-r)^{T}\left(R \widehat{W} \widehat{V}^{-1} \widehat{W} R^{T}\right)^{-1}(R \widehat{\theta}-r) \stackrel{d}{\longrightarrow} \chi^{2}(v),
$$

where $\chi^{2}(v)$ indicates a chi-squared random variable with $v$ degrees of freedom. 
Theorem 5 suggests that the asymptotic normality of $\widehat{\theta}$ induces a Wald-type statistic that follows asymptotically $\chi^{2}$ distribution under the null hypothesis. We can test the null hypothesis $H_{0}$ by setting

$$
R=\left(\begin{array}{cccccc}
1 & 0 & 0 & -1 & 0 & 0 \\
0 & 1 & 0 & 0 & -1 & 0 \\
0 & 0 & 1 & 0 & 0 & -1
\end{array}\right) \quad \text { and } r=(0,0,0)^{T}
$$

Then the Wald-type statistic $T_{N, M}$ follows $\chi^{2}(3)$. In the empirical study, we reveal the state heterogeneity in S\&P 500 index volatility by conducting the proposed Wald-type test. Details can be found in Section 5 .

Remark 7. Theorems 25 are established based on the decomposition of expected integrated volatility in Theorem 1 (a),

$$
\int_{n-1}^{n} \sigma_{i, t}^{2} d t=h_{i, n}(\theta)+\xi_{i, n} \text { a.s. }
$$

and their results can be established for any instantaneous volatility process that satisfies Theorem 1(a). The SG-Itô model is one of the examples.

\section{$5 \quad$ Numerical studies}

\subsection{Simulation studies}

To evaluate the relevance of asymptotic theories, we conducted simulation studies. We first simulated the log price process and assessed the finite sample performance of the suggested estimator $\widehat{\theta}$. The $\log$ stock price $X_{t_{n, m}}$ for $t_{n, m}=n-1+m / M$ was generated from the SG-Itô model in Definition 1 with the following form:

$$
\begin{aligned}
& d X_{t}=\mu d t+\sigma_{t} d B_{t}, \quad \sigma_{t}=\left(1-s_{n}\right) \sigma_{1, t}+s_{n} \sigma_{2, t}, \\
& \sigma_{i, t}^{2}=\sigma_{[t]}^{2}+(t-[t])\left\{\omega_{0, i}+\left(\gamma_{0, i}-1\right) \sigma_{[t]}^{2}\right\}+\beta_{0, i}\left(X_{t}-X_{[t]}-(t-[t]) \mu\right)^{2}, \\
& s_{n}=\mathbb{1}\left\{\left(X_{n-1}-X_{n-2}\right)<0\right\},
\end{aligned}
$$


where $\mathbb{1}\{\cdot\}$ is an indicator function and $\theta_{0}=\left(\omega_{0,1}, \omega_{0,2}, \beta_{0,1}, \beta_{0,2}, \gamma_{0,1}, \gamma_{0,2}\right)$ is the true model parameter. To capture the leverage effect, we set $s_{n}=1$ if the previous day return is negative and $s_{n}=0$ otherwise. The price process was generated under the null and alternative hypothesis, respectively. Under the null hypothesis, the true parameter set is given by $\theta_{0}=$ $(0.15,0.15,0.2,0.2,0.1,0.1)$, whereas under the alternative hypothesis, the true parameter set is given by $\theta_{0}=(0.15,0.165,0.2,0.22,0.1,0.11)$. We set initial price $X_{0}=10$, initial instantaneous volatility $\sigma_{i, 0}^{2}=\frac{\omega_{i}^{h}\left(1-\beta_{i}^{h}-\gamma_{i}\right)+\beta_{i} \omega_{i}^{h}}{\left(1-\beta_{i}^{h}-\gamma_{i}\right)\left(1-\gamma_{i}\right)}$, and $\mu=0$. We chose $N=1000$ and $M=23400$, corresponding to the stock price observed every second during four years. The Euler scheme was applied to discritize continuous-time processes. The observed price $Y_{t_{n, m}}$ was calculated as the sum of the true $\log$ price $X_{t_{n, m}}$ and the micro-structure noise $\epsilon_{t_{n, m}}$, where $X_{t_{n, m}}$ was generated from Equation (5.1) and $\epsilon_{t_{n, m}}$ was generated from i.i.d. normal distribution with mean zero and standard deviation $\sigma_{\epsilon}=0.01$. For realized volatility estimator, we employed the pre-averaging method Christensen et al., 2010; Jacod et al., 2009), presented as follows:

$$
\begin{aligned}
& R V_{n}=\frac{1}{\phi_{K}(f)} \frac{M}{M-K} \sum_{k=1}^{M-K+1}\left(\bar{Y}\left(t_{k}\right)^{2}-\frac{1}{2} \widehat{Y}_{k}\right), \\
& \bar{Y}\left(t_{k}\right)=\sum_{i=1}^{K-1} f\left(\frac{i}{K}\right)\left[Y_{t, k+i}-Y_{t, k+i-1}\right], \phi_{K}(f)=\sum_{i=1}^{K} f\left(\frac{i}{K}\right)^{2}, \\
& \widehat{Y}_{k}=\sum_{i=1}^{K}\left(f\left(\frac{i}{K}\right)-f\left(\frac{i-1}{K}\right)\right)^{2}\left(Y_{t, k+i}-Y_{t, k+i-1}\right)^{2},
\end{aligned}
$$

where $K=[\sqrt{M}]$ is the tuning parameter that determines the number of observations used for the pre-averaging step and $f(x)=\min (x, 1-x)$. Using the generated stock prices, we estimated the realized volatility and calculated $\widehat{\theta}$ using the QMLE method in Section 3 . The simulation procedure was repeated 1000 times.

We first examined the effect of period and frequency of the data on parameter estimation. The accuracy of model parameter estimation is expected to be improved by longer period and higher frequency data. To verify this, we generated additional data sets by resampling the entire data. Specifically, we collected first 250, 500, 750, and 1000-day data for $N=250,500,750$, and 1000, 
respectively. We also collected one of every 60, 10, and 5 data in each day corresponding to 1minute $(M=390), 10$-second $(M=2340)$, and 5 -second data $(M=4680)$, respectively. Figure 1 provides mean squared errors (MSEs) of the estimator $\widehat{\theta}$ for varying $N$ and $M$. From Figure 1 . we find that the use of longer period and higher frequency data significantly improves estimation performance, which supports the theoretical findings in Section 3 .

\section{[Figure 1 inserted about here]}

To investigate the advantage of considering state heterogeneity in the model, we compared the prediction performance of the SG-Itô model with that of the existing volatility models including $\operatorname{GARCH}(1,1)$, RS-GARCH$(1,1)$, and unified GARCH-Itô models. Conditional daily volatility processes of the GARCH and unified GARCH-Itô models are presented as follows:

$$
\begin{aligned}
& h_{n}\left(\theta^{L}\right)=\omega^{L}+\gamma^{L} h_{n-1}\left(\theta^{L}\right)+\beta^{L} Z_{n-1}^{2}, \\
& h_{n}\left(\theta^{g}\right)=\omega^{g *}+\gamma^{g} h_{n-1}\left(\theta^{g}\right)+\beta^{g *} Z_{n-1}^{2},
\end{aligned}
$$

where $\theta^{L}=\left(\omega^{L}, \gamma^{L}, \beta^{L}\right)$ is the model parameter of the GARCH model, and $\theta^{g}$ is the model parameter of the unified GARCH-Itô model described in Appendix A.2. The RS-GARCH(1,1) model is illustrated in Equation (2.1). The QMLE of $\theta^{L}$ and $\theta^{s, L}$ were obtained as in Equation (3.2), whereas that of the unified GARCH-Itô parameter was obtained by maximizing Equation (3.1). Note that in parameter estimation, the discrete-time models employ daily return square, whereas the continuous-time models employ daily realized volatility estimates. To evaluate oneday ahead out-of-sample prediction performances, we calculated the mean squared prediction error (MSPE) of each model as follows:

$$
M S P E=\frac{1}{d} \sum_{n=1}^{d}\left(R V_{n}-V_{n}\right)^{2},
$$

where $V_{n}$ is a fitted variance generated from each volatility model, $d$ is the length of prediction window, and the length of both estimation and prediction windows are set to 500. Figure 2 draws 
the log MSPEs of the GARCH, RS-GARCH, unified GARCH-Itô, and SG-Itô models under the null and alternative hypotheses. In the comparison of the discrete-time models (i.e., GARCH and RS-GARCH) and continuous-time models (i.e., unified GARCH-Itô and SG-Itô), we find that the continuous-time models perform better. This may be because the discrete-time models need relatively long time periods to obtain consistent estimators, whereas the continuous-time models can estimate the model parameters well in short time period. This improvement stems from the efficiency of the realized volatility estimator. In the comparison of the unified GARCH-Itô and SG-Itô models, under the null hypothesis, the unified GARCH-Itô model performs better. This is because under the null hypothesis, the unified GARCH-Itô model is true, so the complexity of the SG-Itô model brings the inefficiency of parameter estimation. On the other hand, the SG-Itô model outperforms under the alternative hypothesis. This may be because the SG-Itô model can deal with the state heterogeneity in volatility dynamics, whereas the unified GARCH-Itô model cannot.

\section{[Figure 2 inserted about here]}

To check the performance of the Wald-type test statistic $T_{N, M}$ developed in Section 4, we investigated the asymptotic convergence of the statistic and conducted size $\alpha$ tests. Figure 3 reports $\chi^{2}$ quantile-quantile plots of the Wald-type statistic $T_{N, M}$ by varying $N, M$ under the null hypothesis. The real line in the figures denotes the best linear fitted line that illustrates perfect $\chi^{2}$ distribution. Figure 3 shows that the Wald-type statistic $T_{N, M}$ gradually closes to the limiting distribution $\chi^{2}$ as $N$ and $M$ increase. Table 1 reports the rejection rate of hypothesis test for significance levels of $0.1,0.05,0.025,0.01$ by varying $N, M$ under the null and alternative hypotheses. In Table 1, we find that under the null hypothesis, the type I error becomes closer to the suggested significance level $\alpha$ as $N$ and $M$ increase. That is, the proposed test procedure satisfies size $\alpha$ tests asymptotically. Under the alternative hypothesis, the power becomes closer to one as $N$ and $M$ increase. These results support the theoretical findings in Section 4.

[Table 1 inserted about here] 


\section{[Figure 3 inserted about here]}

\subsection{Empirical studies}

In the empirical study, we examined the volatility process of S\&P 500 index return under the SGItô framework. We used intraday S\&P 500 index data from 9:30 a.m. to 4:00 p.m., spanning from January 2, 2015, to December 31, $2018(N=998)$, provided by Chicago Board of Exchange. Before July 23, 2015, data sampling frequency varied from one to three seconds, so the number of intraday data $M_{n}$ varied from 10,000 to 23,400 . After July $24,2015, M_{n}$ was fixed to 23,400 except for early closing days. We constructed daily pre-averaging realized volatility estimates using intraday index data.

For the SG-Itô model, the state process plays a prominent role in model specification. In this empirical study, we considered seven state processes that are known to affect financial volatility and defined models (i)-(vii) corresponding to each state variable. The first two models are related to market returns. These models deal with the negative correlation between financial return and future volatility, which is called the leverage effect (Black, 1976, Christie, 1982, Figlewski and Wang, 2000. Tauchen et al., 1996). For the market return states, we calculated (i) open-to-close returns for the the previous day market return and (ii) close-to-open returns for the overnight return. We assigned $s_{n}=1$ if (i) the open-to-close return was included in the lowest three deciles and (ii) the overnight return was negative, respectively, and $s_{n}=0$ otherwise. Note that models (i) and (ii) incorporate the GJR-GARCH model. Second, we considered the Chinese stock market information in model (iii). As the second-largest economy in the world, Chinese economy and their stock market may comove with that of the U.S. Moreover, the Chinese stock market indices contain information for the non-trading hours in the U.S. stock market. Thus, we suppose that the Chinese stock market movement affects the U.S. stock market volatility. We assigned $s_{n}=1$ if the Hang Seng index return was included in the lowest three deciles and $s_{n}=0$ otherwise. Third, we considered the day-of-week seasonality in the financial market, especially on pre- and post-holiday Abraham and Ikenberry, 1994; French, 1980; Lakonishok and Maberly, 1990; Miller, 1988). Specifically, for models (iv) and 
(v), we constructed pre- and post-holiday indicators using NYSE holiday data and assigned $s_{n}=1$ on day (iv) before and (v) after NYSE holidays, including weekends, respectively, and $s_{n}=0$ otherwise. Fourth, we considered trading volume and investor attention. Previous studies showed that they are positively correlated with financial volatility (Andrei and Hasler, 2014; Copeland, 1976; Jennings et al., 1981; Lamoureux and Lastrapes, 1990a, 1994). We measured trading volume and investor attention together with abnormal trading volume abtv, calculated by the aggregate market daily dollar volume divided by the sum of recent 20 days dollar volume (Barber and Odean, 2007). The large abtv presents the day of high trading volume and high investor attention. For the model (vi), we assigned $s_{n}=1$ if the day with abtv was greater than average, and $s_{n}=0$ otherwise. Finally, we adopted an illiquidity measure to proxy the bid-ask based aggregate market illiquidity (Chen et al. 2018, Wang and Yau, 2000). The Corwin and Schultz 2012, CS) measure gauges the illiquidity of individual stocks based on daily high-low spread as follows:

$$
\begin{aligned}
& c s=\frac{2\left(e^{\delta}-1\right)}{1+e^{\delta}} \\
& \delta=\frac{\sqrt{2 \tau}-\sqrt{\tau}}{3-2 \sqrt{2}}-\sqrt{\frac{\rho}{3-2 \sqrt{2}}}, \tau=\left[\log \left(\frac{H_{t-1}}{L_{t-1}}\right)\right]^{2}+\left[\log \left(\frac{H_{t}}{L_{t}}\right)\right]^{2}, \rho=\left[\log \left(\frac{H_{t-1, t}}{L_{t-1, t}}\right)\right]^{2},
\end{aligned}
$$

where $H_{t-1, t}$ and $L_{t-1, t}$ are high and low price over days $t-1$ and $t$, respectively. For model (vii), we calculated firm-specific CS measures and value-weighted them to construct the aggregate market illiquidity measure vwcs. We assigned $s_{n}=1$ if vwcs was in the highest three deciles, which denoted an illiquid day, and $s_{n}=0$ otherwise.

Table 2 reports the SG-Itô model parameter estimation and hypothesis test results. The parameter estimates provide some interesting features of volatility processes. For example, $\omega_{2}$ and $\gamma_{2}$ of the models (i), (ii), and (iii) are significantly higher than $\omega_{1}$ and $\gamma_{1}$, respectively, which means that the volatility is generally greater after negative return shock and their clustering become strengthened. In particular, the greater $\beta_{2}$ of the model (ii) (0.212) than the model (i) (0.136) may suggest that the market volatility is more sensitive to overnight shocks than the previous day market returns. Parameter estimates of model (vi) suggest that the impacts of the previous volatility and 
return shocks on the present volatility increase with heavy tradings.

\section{[Table 2 inserted about here]}

The results of hypothesis testing suggest that the null hypothesis $H_{0}:\left\{\omega_{1}=\omega_{2}, \gamma_{1}=\gamma_{2}, \beta_{1}=\right.$ $\left.\beta_{2}\right\}$ is rejected at the $1 \%$ level for models (i)-(iv), (vi), and (vii). This implies that the volatility process is distinguished from the homogeneous volatility process when (i) previous day open-toclose return is significantly low, (ii) overnight return is negative, (iii) Hang Seng index return is significantly low, (iv) investors prepare for upcoming holidays, (vi) aggregate trading volume is abnormally high, and (vii) the market is illiquid. These results are in line with existing studies. For example, Braun et al. (1995), Carr and Wu (2017), and Kim and Kon (1994) demonstrated that market volatility is significantly increased by negative return shocks. Ahoniemi and Lanne (2013), Ahoniemi et al. (2016), and Tsiakas (2008) reveal that the overnight information significantly affect the stock market dynamics and help forecast asset volatility. In particular, the Asian stock markets possibly reflect overnight information of the U.S. stock market because of their time lag (Taylor, 2007). We provide the evidence of close relationship between the U.S. and Chinese stock markets. Gallant et al. (1992), Kambouroudis and McMillan (2016), and Karpoff (1987) showed that aggregate trading volume is positively related to future market volatility. The existence of day-of-week and holiday effect are remain up for debate. Berument and Kiymaz (2001) and Kiymaz and Berument (2003) showed the existence of day-of-week effect on market volatility, whereas Birru (2018) claimed that the effect has disappeared on an aggregate level. The hypothesis testing results for models (iv) and (v) suggest that the pre-holiday effect on the market volatility process may exist, whereas the post-holiday effect has disappeared.

Table 3 shows the integrated form of the SG-Itô model parameter estimates described in Theorem 1(b). Table 4 presents parameter estimates of $\operatorname{GARCH}(1,1)$, unified GARCH-Itô (i.e., $\omega^{g *}$, $\left.\gamma^{g *}, \beta^{g *}\right)$, and RS-GARCH(1,1) models. The integrated form of the SG-Itô model parameters can be interpreted similarly to the RS-GARCH(1,1) model parameters. For example, the large $\beta_{12}^{h}$ and $\beta_{22}^{h}$ of the model (ii) in Table 3 may suggest that daily integrated volatility is significantly affected by market return after negative overnight return shocks. This is in line with the large $\beta_{2}^{L}$ of the 
RS-GARCH model (ii) in Table 4 .

[Table 3 inserted about here]

[Table 4 inserted about here]

To investigate the efficiency of adopting the high-frequency data, we estimated the SG-Itô model parameters and Wald-type statistics using low-frequency data only. We estimated parameter

estimates $\widehat{\theta}^{L}$ with low-frequency data using the Equation 3.2 . Then we can calculate the Waldtype statistic for $\widehat{\theta}^{L}$, as follows:

$$
T_{N}=N\left(R \widehat{\theta}^{L}-r\right)^{T}\left(R \widehat{W}\left(\widehat{V}^{L}\right)^{-1} \widehat{W} R^{T}\right)^{-1}\left(R \widehat{\theta}^{L}-r\right) \stackrel{d}{\longrightarrow} \chi^{2}(v)
$$

where

$$
\widehat{V}^{L}=\frac{1}{4 N} \sum_{n=1}^{N}\left[\left.\frac{\partial h_{n}(\theta)}{\partial \theta}\left[\frac{\partial h_{n}(\theta)}{\partial \theta}\right]^{T}\right|_{\theta=\widehat{\theta}} h_{n}(\widehat{\theta})^{-4}\left(\zeta_{n}^{2}-h_{n}(\widehat{\theta})\right)^{2}\right]
$$

Table 5 reports SG-Itô model parameter estimation and hypothesis test results based on lowfrequency data only. We find that standard errors of parameters are significantly increased compared with the results in Table 2 , and, accordingly, most of the $\omega$ s and $\beta$ s are not significant at the $1 \%$ level anymore. Moreover, the Wald-type test fails to detect the state heterogeneity in models (ii)-(iv), and the significance of rejection has reduced for models (i) and (vi) as well. These results may imply that the relatively short period of low-frequency data may not contain sufficient information and fail to capture low-frequency volatility dynamics. From the results, we can conclude that the use of high-frequency data helps to analyze low-frequency dynamics for relatively shorttime-period data, so it would be more robust to the structural break issue. These findings support our hypotheses of existence of state heterogeneity and efficiency of using high-frequency data to examine low-frequency market dynamics.

[Table 5 inserted about here] 
Foregoing results indicate the existence of state heterogeneity in the volatility process for wellknown state variables. This may imply that the volatility model considering state heterogeneity would produce better volatility estimates and forecasts. To compare the prediction performance of representative volatility models, we implemented the one-day-ahead out-of-sample volatility prediction and calculated mean absolute percentage error (MAPE), presented as follows:

$$
M A P E=\frac{100}{d} \sum_{n=1}^{d}\left|\frac{R V_{n}-V_{n}}{R V_{n}}\right|,
$$

where $d$ is the length of prediction window and $V_{n}$ is a fitted variance generated from each volatility model. The estimation window is 750 days with the prediction period spanning from December 22, 2017, to December 31, 2018 (248 days). The benchmarks are unified GARCH-Itô, RS-GARCH(1,1), and GARCH(1,1) models and the model specifications are presented in Section 5.1 . We also consider heterogeneous auto-regressive (HAR) model of Corsi (2009) as an additional benchmark. On the one hand, state variable $s_{n}$ is available at the beginning of day $n$ for models (i) previous day open-to-close return, (ii) overnight return, (iii) Hang Seng index return, (iv) pre-holiday, and (v) post-holiday. On the other hand, $s_{n}$ is not observed until the end of day $n$ for the models (vi) abnormal trading volume and (vii) market illiquidity, so we have to utilize a state transition probability as in Proposition 1. To obtain state transition probability, we simply assumed time-persistent state transition probability and calculated the portion of transition from state $j$ to $i$ for $p_{i j}$. The estimation of state transition probability significantly affects prediction performance, but we leave a more elaborate probability inference for further research. Table 6 reports out-of-sample prediction results measured by MAPEs. The results suggest that continuous-time models (SG-Itô and unified GARCH-Itô) performs better than discrete-time models (GARCH and RS-GARCH) and the HAR model and that state heterogeneity models (SG-Itô and RS-GARCH) are superior to state-homogeneous models (GARCH and unified GARCH-Itô) in general. Thus, the continuous and state heterogeneous SG-Itô model shows outstanding performance compared to the others. In particular, the MAPE improvement of the SG-Itô model is the greatest in model (ii), in which the state heterogeneity was the greatest, whereas the improvement seems insignificant in model (v), 
in which state heterogeneity was not detected. For models (vi) and (vii), although we employed the simple procedure to estimate transition probability, the prediction performance of the SG-Itô model is similar to or even better than that of the benchmark models.

\section{[Table 6 inserted about here]}

\section{Conclusions}

State heterogeneity in financial volatility has widely been discussed as a representative market characteristic. This study hypothesizes that there exists state heterogeneity in financial volatility and use of high-frequency data facilitates analyzing it. To test the hypothesis, we proposed a novel volatility model whose instantaneous volatility has a continuous-time process and that evolves depending on the discrete state process. Through the model, this study provides a mathematical background to apply high-quality realized volatility estimators to the study of discrete-time state heterogeneity volatility frameworks. Along with the model, we construct a Wald-type hypothesis testing procedure to test our hypothesis. Through hypothesis testing, we verify the existence of leverage, investor attention, market illiquidity, stock market comovement, and post-holiday effect in S\&P 500 index volatility. The statistical test based on low-frequency data only, however, does not catch these effects well.

In this paper, our focus is to test the given exogenous state. However, in practice, how to define the state process is an important but difficult question. Fortunately, the proposed SG-Itô diffusion process is not affected by the state process, so it is easy to incorporate any state process in the SG-Itô process structure. Thus, studying state processes based on the high-frequency financial data is a promising direction for future research. 


\section{A Appendix}

\section{A.1 Instantaneous volatility}

Under the SG-Itô framework, the instantaneous volatility at integer time point $n$ can be presented as the linear function of $\sigma_{n-1}^{2}$ and daily return square as follows:

$$
\begin{aligned}
\sigma_{n}^{2} & =\left(1-s_{n}\right) \sigma_{1, n}^{2}+s_{n} \sigma_{2, n}^{2} \\
& =\left(1-s_{n}\right)\left(\omega_{1}+\beta_{1} Z_{1, n}^{2}\right)+s_{n}\left(\omega_{2}+\beta_{2} Z_{2, n}^{2}\right)+\left(\left(1-s_{n}\right) \gamma_{1}+s_{n} \gamma_{2}\right) \sigma_{n-1}^{2}
\end{aligned}
$$

Then we can express instantaneous volatility at integer time point as the infinite sum of $Z_{i, n}$ 's using a recursive relationship. Let $D_{n}(k)=\left[\left(1-s_{n+1-k}\right) \gamma_{1}+s_{n+1-k} \gamma_{2}\right] D_{n}(k-1)$ and $D_{n}(0)=1$. Then $D_{n}(k)=\prod_{i=0}^{k-1}\left[\left(1-s_{n-i}\right) \gamma_{1}+s_{n-i} \gamma_{2}\right]$ and we have

$$
\begin{aligned}
\sigma_{n}^{2}= & D_{n}(0)\left\{\left(1-s_{n}\right)\left(\omega_{1}+\beta_{1} Z_{1, n}^{2}\right)+s_{n}\left(\omega_{2}+\beta_{2} Z_{2, n}^{2}\right)\right\}+D_{n}(1) \sigma_{n-1}^{2} \\
= & D_{n}(0)\left\{\left(1-s_{n}\right)\left(\omega_{1}+\beta_{1} Z_{1, n}^{2}\right)+s_{n}\left(\omega_{2}+\beta_{2} Z_{2, n}^{2}\right)\right\} \\
& +D_{n}(1)\left\{\left(1-s_{n-1}\right)\left(\omega_{1}+\beta_{1} Z_{1, n-1}^{2}\right)+s_{n-1}\left(\omega_{2}+\beta_{2} Z_{2, n-1}^{2}\right)\right\}+D_{n}(2) \sigma_{n-2}^{2} \\
= & \sum_{i=0}^{k-1} D_{n}(i)\left\{\left(1-s_{n-i}\right)\left(\omega_{1}+\beta_{1} Z_{1, n-i}^{2}\right)+s_{n-i}\left(\omega_{2}+\beta_{2} Z_{2, n-i}^{2}\right)\right\}+D_{n}(k) \sigma_{n-k}^{2} \\
= & \sum_{i=0}^{\infty} D_{n}(i)\left\{\left(1-s_{n-i}\right)\left(\omega_{1}+\beta_{1} Z_{1, n-i}^{2}\right)+s_{n-i}\left(\omega_{2}+\beta_{2} Z_{2, n-i}^{2}\right)\right\} \text { a.s. }
\end{aligned}
$$

Note that $D_{n}(k) \leq\left(\gamma_{u}^{h}\right)^{k}, \sigma_{n}^{2}$ satisfies following inequality:

$$
\sigma_{n}^{2} \leq \sum_{i=0}^{\infty}\left(\gamma_{u}^{h}\right)^{i}\left(\omega_{u}^{h}+\beta_{u}^{h} Z_{n-i}^{2}\right)=\frac{\omega_{u}^{h}}{1-\gamma_{u}^{h}}+\beta_{u}^{h} \sum_{i=0}^{\infty}\left(\gamma_{u}^{h}\right)^{i} Z_{n-i}^{2}
$$

Then, by Assumption 1(d), we can easily show the existence of the infinite sum in A.1. 


\section{A.2 Connection with the GARCH-Itô model}

We can show that when the states are homogeneous $\left(s_{n}=s_{n-1}=\cdots=s_{1}\right)$, the SG-Itô model returns to the unified GARCH-Itô model (Kim and Wang, 2016). That is, the unified GARCH-Itô model is a special example of the SG-Itô model. The unified GARCH-Itô model can be presented as follows:

$$
\begin{aligned}
& d X_{t}=\mu d t+\sigma_{t} d B_{t}, \\
& \sigma_{t}^{2}=\sigma_{n-1}^{2}+(t-n+1)\left\{\omega^{g}+\left(\gamma^{g}-1\right) \sigma_{n-1}^{2}\right\}+\beta^{g}\left(\int_{n-1}^{t} \sigma_{s} d B_{s}\right)^{2},
\end{aligned}
$$

where $\theta^{g}=\left(\omega^{g}, \beta^{g}, \gamma^{g}\right)$ are model parameters. Let us assume $s_{n}=1$ for all $n \in \mathbb{N}$. Then, by Equation (A.1), the instantaneous volatility under the SG-Itô model can be presented as follows:

$$
\sigma_{n}^{2}=\sum_{i=0}^{\infty} \gamma_{1}^{i}\left(\omega_{1}+\beta_{1} Z_{n-i}^{2}\right)=\frac{\omega_{1}}{1-\gamma_{1}}+\beta_{1} \sum_{i=0}^{\infty} \gamma_{1}^{i} Z_{n-i}^{2}
$$

For $h_{n}(\theta)$, we have

$$
\begin{aligned}
h_{n}(\theta) & =\omega_{11}^{h}+\gamma_{11}^{h} h_{n-1}(\theta)+\beta_{11}^{h} Z_{n-1}^{2} \\
& =\omega^{g *}+\gamma^{g} h_{n-1}(\theta)+\beta^{g *} Z_{n-1}^{2},
\end{aligned}
$$

where $\omega^{g *}=\omega^{g}\left(\beta^{g}\right)^{-1}\left(e^{\beta^{g}}-1\right)$ and $\beta^{g *}=\left(\beta^{g}\right)^{-1}\left(\gamma^{g}-1\right)\left(e^{\beta^{g}}-1-\beta^{g}\right)+e^{\beta^{g}}-1$.

\section{A.3 Proof of Theorem 1}

Proof. Proof of Theorem 1 Consider $(a)$. By Itô's lemma, we have

$$
\begin{aligned}
R_{1}(k)= & \int_{n-1}^{n} \frac{(n-t)^{k}}{k !} \sigma_{1, t}^{2} d t \\
= & \int_{n-1}^{n} \frac{(n-t)^{k}}{k !}\left[\sigma_{n-1}^{2}+(t-n+1)\left\{\omega_{1}+\left(\gamma_{1}-1\right) \sigma_{n-1}^{2}\right\}\right] d t \\
& +\beta_{1} \int_{n-1}^{n} \frac{(n-t)^{k}}{k !}\left(\int_{n-1}^{t} \sigma_{1, s} d B_{s}\right)^{2} d t
\end{aligned}
$$




$$
\begin{aligned}
= & \frac{1}{(k+2) !}\left[\omega_{1}+\left(\gamma_{1}+k+1\right) \sigma_{n-1}^{2}\right] \\
& +2 \beta_{1} \int_{n-1}^{n} \frac{(n-t)^{k+1}}{(k+1) !}\left(\int_{n-1}^{t} \sigma_{1, s} d B_{s}\right) \sigma_{1, t} d B_{t}+\beta_{1} R_{1}(k+1) .
\end{aligned}
$$

Then we have

$\int_{n-1}^{n} \sigma_{1, t}^{2} d t=R_{1}(0)=\beta_{1}^{-2}\left(e^{\beta_{1}}-1-\beta_{1}\right) \omega_{1}+\left[\left(\gamma_{1}-1\right) \beta_{1}^{-2}\left(e^{\beta_{1}}-1-\beta_{1}\right)+\beta_{1}^{-1}\left(e^{\beta_{1}}-1\right)\right] \sigma_{n-1}^{2}+\xi_{1, n}$,

where $\xi_{1, n}=2 \int_{n-1}^{n}\left(e^{(n-t) \beta_{1}}-1\right) \int_{n-1}^{t} \sigma_{1, s} d B_{s} \sigma_{1, t} d B_{t}$. We can calculate $\int_{n-1}^{n} \sigma_{2, t}^{2} d t$ in the same way. Then integrated volatility under the SG-Itô framework can be expressed as a function of $\left\{\mathcal{F}_{n-1}^{x, L}, \mathcal{F}_{n-1}^{s}\right\}$-adapted process and the martingale difference as follows:

$$
\begin{aligned}
& \int_{n-1}^{n} \sigma_{i, t}^{2} d t=h_{i, n}(\theta)+\xi_{i, n} \\
& E\left[\int_{n-1}^{n} \sigma_{i, t}^{2} d t \mid \mathcal{F}_{n-1}\right]=h_{i, n}(\theta)=H_{c, i}(\theta)+H_{\beta, i}(\theta) \sigma_{n-1}^{2} \text { a.s. }
\end{aligned}
$$

Consider $(b)$. By the result of $(a)$, we have

$$
\begin{aligned}
E\left[\int_{n-1}^{n} \sigma_{t} d t \mid \mathcal{F}_{n-1}\right] & =h_{n}(\theta)=\left(1-s_{n}\right) h_{1, n}(\theta)+s_{n} h_{2, n}(\theta) \\
& =\left(1-s_{n}\right)\left(H_{c, 1}(\theta)+H_{\beta, 1}(\theta) \sigma_{n-1}^{2}\right)+s_{n}\left(H_{c, 2}(\theta)+H_{\beta, 2}(\theta) \sigma_{n-1}^{2}\right) \\
& =H_{c, n}^{w}(\theta)+H_{\beta, n}^{w}(\theta) \sigma_{n-1}^{2},
\end{aligned}
$$

where $H_{c, n}^{w}(\theta)=\left(1-s_{n}\right) H_{c, 1}(\theta)+s_{n} H_{c, 2}(\theta)$ and $H_{\beta, n}^{w}(\theta)=\left(1-s_{n}\right) H_{\beta, 1}(\theta)+s_{n} H_{\beta, 2}(\theta)$. By Equation A.1., we have

$$
\begin{aligned}
& h_{n}(\theta)=H_{c, n}^{w}(\theta)+H_{\beta, n}^{w}(\theta) \sigma_{n-1}^{2} \\
& =H_{c, n}^{w}(\theta)+H_{\beta, n}^{w}(\theta) \sum_{i=0}^{\infty} D_{n-1}(i)\left\{\left(1-s_{n-1-i}\right)\left(\omega_{1}+\beta_{1} Z_{1, n-1-i}^{2}\right)+s_{n-i}\left(\omega_{2}+\beta_{2} Z_{2, n-1-i}^{2}\right)\right\} \\
& =H_{c, n}^{w}(\theta)+H_{\beta, n}^{w}(\theta) \sum_{i=1}^{\infty} D_{n-1}(i)\left\{\left(1-s_{n-1-i}\right)\left(\omega_{1}+\beta_{1} Z_{1, n-1-i}^{2}\right)+s_{n-i}\left(\omega_{2}+\beta_{2} Z_{2, n-1-i}^{2}\right)\right\}
\end{aligned}
$$




$$
\begin{aligned}
& +H_{\beta, n}^{w}(\theta)\left\{\left(1-s_{n-1}\right)\left(\omega_{1}+\beta_{1} Z_{1, n-1}^{2}\right)+s_{n-1}\left(\omega_{2}+\beta_{2} Z_{2, n-1}^{2}\right)\right\} \\
= & H_{c, n}^{w}(\theta)+H_{\beta, n}^{w}(\theta) D_{n-1}(1) \sigma_{n-2}^{2}+H_{\beta, n}^{w}(\theta)\left\{\left(1-s_{n-1}\right)\left(\omega_{1}+\beta_{1} Z_{1, n-1}^{2}\right)+s_{n-1}\left(\omega_{2}+\beta_{2} Z_{2, n-1}^{2}\right)\right\} .
\end{aligned}
$$

Thus, we have

$$
h_{n}(\theta)=\omega_{n}^{w}+\gamma_{n}^{w} h_{n-1}(\theta)+\beta_{n}^{w} Z_{n-1}^{2},
$$

where $\omega_{n}^{w}=H_{c, n}^{w}(\theta)+H_{\beta, n}^{w}(\theta)\left\{\left(1-s_{n-1}\right) \omega_{1}+s_{n-1} \omega_{2}\right\}-\gamma_{n}^{w} H_{c, n-1}^{\omega}(\theta), \gamma_{n}^{w}=\frac{D_{n-1}(1) H_{\beta, n}^{\omega}(\theta)}{H_{\beta, n-1}^{\omega}(\theta)}, \beta_{n}^{w}=$ $H_{\beta, n}^{w}(\theta)\left\{\left(1-s_{n-1}\right) \beta_{1}+s_{n-1} \beta_{2}\right\}$. Then we can easily show

$$
\begin{aligned}
h_{n}(\theta)= & s_{11, n}\left(\omega_{11}^{h}+\beta_{11}^{h} h_{n-1}(\theta)+\gamma_{11}^{h} Z_{n-1}^{2}\right)+s_{12, n}\left(\omega_{12}^{h}+\beta_{12}^{h} h_{n-1}(\theta)+\gamma_{12}^{h} Z_{n-1}^{2}\right) \\
& +s_{21, n}\left(\omega_{21}^{h}+\beta_{21}^{h} h_{n-1}(\theta)+\gamma_{21}^{h} Z_{n-1}^{2}\right)+s_{22, n}\left(\omega_{22}^{h}+\beta_{22}^{h} h_{n-1}(\theta)+\gamma_{22}^{h} Z_{n-1}^{2}\right) .
\end{aligned}
$$

\section{A.4 Proof of asymptotic theories}

This section provides proofs of asymptotic theories presented in Section 3 . First Lemma 1 shows that the impact of the initial value is asymptotically negligible by showing that the impact of initial value on $h_{n}(\theta)$ is exponentially decaying. Accordingly, the difference between the quasi-likelihood functions with true and arbitrary value decays faster than $O_{p}\left(N^{-1}\right)$.

\section{A.4.1 Initial value}

Lemma 1. Under Assumption $1(a)$, we have for any $\vartheta=O_{p}(1)$ and $n \in \mathbb{N}, \mid h_{n}\left(\theta_{0}, \sigma_{0}^{2}\right)-$ $h_{n}\left(\theta_{0}, \vartheta\right) \mid=O_{p}\left(\left(\gamma_{u}^{h}\right)^{n-1}\right)$.

Proof. Proof. Simple algebraic manipulations provide

$$
h_{n}\left(\theta_{0}, \sigma_{0}^{2}\right)-h_{n}\left(\theta_{0}, \vartheta\right)=\prod_{k=1}^{n-1} \gamma_{n-k+1}^{w}\left(h_{1}\left(\theta_{0}, \sigma_{0}^{2}\right)-h_{1}\left(\theta_{0}, \vartheta\right)\right)
$$




$$
\leq\left(\gamma_{u}^{h}\right)^{n-1} H_{\beta, 1}^{w}(\theta)\left(\sigma_{0}^{2}-\vartheta\right)=O_{p}\left(\left(\gamma_{u}^{h}\right)^{n-1}\right)
$$

Thus, as $N \rightarrow \infty$, the difference between $\sigma_{0}^{2}$ and $\vartheta$ become negligible.

\section{A.4.2 Proof of Theorem 2}

For given $\left\{s_{n}\right\}$, we define the log likelihood functions and their derivatives as follows:

$$
\begin{aligned}
& \widehat{Q}_{N, M}(\theta)=-\frac{1}{2 N} \sum_{n=1}^{N}\left[\log \left(h_{n}(\theta)\right)+\frac{R V_{n}}{h_{n}(\theta)}\right]=-\frac{1}{2 N} \sum_{n=1}^{N} \widehat{q}_{N, M}(\theta), \\
& \widehat{S}_{N, M}(\theta)=\frac{\partial \widehat{Q}_{N, M}(\theta)}{\partial \theta}, \quad \widehat{\theta}=\underset{\theta \in \Theta}{\operatorname{argmax}} \widehat{Q}_{N, M}(\theta), \\
& \widetilde{Q}_{N}(\theta)=-\frac{1}{2 N} \sum_{n=1}^{N}\left[\log \left(h_{n}(\theta)\right)+\frac{\int_{n-1}^{n} \sigma_{t}^{2} d t}{h_{n}(\theta)}\right], \quad \widetilde{S}_{N}(\theta)=\frac{\partial \widetilde{Q}_{N}(\theta)}{\partial \theta}, \\
& Q_{N}(\theta)=-\frac{1}{2 N} \sum_{n=1}^{N}\left[\log \left(h_{n}(\theta)\right)+\frac{h_{n}\left(\theta_{0}\right)}{h_{n}(\theta)}\right], \quad S_{N}(\theta)=\frac{\partial Q_{N}(\theta)}{\partial \theta} .
\end{aligned}
$$

We denote derivatives of function $g$ at $x^{*}$ by $\frac{\partial g\left(x^{*}\right)}{\partial x}=\left.\frac{\partial g(x)}{\partial x}\right|_{x=x^{*}}$. Note that in Assumption 1 (a), we defined upper and lower bounds of $\theta$ and $\theta^{h}$.

Lemma 2. Under Assumption 1(a), we have

(a) $\sup _{n \in \mathbb{N}} E\left[Z_{n}^{2}\right] \leq \frac{\omega_{u}^{h}}{1-\beta_{u}^{h}-\gamma_{u}^{h}}+E\left[h_{1}\left(\theta_{0}\right)\right]<\infty$, and $\sup _{n \in \mathbb{N}} E\left[\sup _{\theta \in \Theta} h_{n}(\theta)\right]<\infty$.

(b) $\xi_{i, n}=\beta_{0, i} \int_{n-1}^{n} e^{(n-t) \beta_{0, i}}\left(Z_{i, t}-Z_{i, n-1}\right)^{2}-\int_{n-1}^{n} e^{(n-t) \beta_{0, i}} \sigma_{i, t}^{2} d t$ a.s. for any $n \in \mathbb{N}$ and $i \in\{1,2\}$.

(c) There exists a neighborhood $B\left(\theta_{0}\right)$ of $\theta_{0}$ such that for any $p \geq 1, \sup _{n \in \mathbb{N}}\left\|\sup _{\theta \in B\left(\theta_{0}\right)} \frac{h_{n}\left(\theta_{0}\right)}{h_{n}(\theta)}\right\|_{L_{p}}<$ $\infty$ and $B\left(\theta_{0}\right) \subset \Theta$.

(d) For any $n \in \mathbb{N}, \sup _{n \in \mathbb{N}}\left\|\sup _{\theta \in B\left(\theta_{0}\right)} \frac{1}{h_{n}(\theta)} \frac{\partial h_{n}(\theta)}{\partial \theta_{j}}\right\|_{L_{p}} \leq C, \sup _{n \in \mathbb{N}}\left\|\sup _{\theta \in B\left(\theta_{0}\right)} \frac{1}{h_{n}(\theta)} \frac{\partial^{2} h_{n}(\theta)}{\partial \theta_{j} \partial \theta_{k}}\right\|_{L_{p}} \leq$ $C$, and $\sup _{n \in \mathbb{N}}\left\|\sup _{\theta \in B\left(\theta_{0}\right)} \frac{1}{h_{n}(\theta)} \frac{\partial^{3} h_{n}(\theta)}{\partial \theta_{j} \partial \theta_{k} \partial \theta_{l}}\right\|_{L_{p}} \leq C$ for any $j, k, l \in\{1,2,3,4,5,6\}$, where $\theta=$ $\left(\theta_{1}, \theta_{2}, \theta_{3}, \theta_{4}, \theta_{5}, \theta_{6}\right)=\left(\omega_{1}, \omega_{2}, \gamma_{1}, \gamma_{2}, \beta_{1}, \beta_{2}\right)$. 
Proof. Proof. For (a), by Equation A.4, we can express daily integrated volatility as infinite sum of $Z_{i, n}$ 's as follows:

$$
\begin{aligned}
h_{n}(\theta) & =\omega_{n}^{w}+\gamma_{n}^{w} h_{n-1}(\theta)+\beta_{n}^{w} Z_{n-1}^{2} \\
& =\sum_{k=1}^{n-1}\left(\omega_{n-k+1}^{w}+\beta_{n-k+1}^{w} Z_{n-k}^{2}\right) \prod_{l=1}^{k-1} \gamma_{n-l+1}^{w}+\prod_{k=1}^{n-1} \gamma_{n-k+1}^{w} h_{1}(\theta) .
\end{aligned}
$$

Using iterative relationship and $\gamma_{n}^{w}+\beta_{n}^{w}<1$, we can show that

$$
\begin{aligned}
E\left[Z_{n}^{2}\right] & =E\left[h_{n}\left(\theta_{0}\right)\right]=\omega_{n}^{w}+\gamma_{n}^{w} E\left[h_{n-1}\left(\theta_{0}\right)\right]+\beta_{n}^{w} E\left[Z_{n-1}^{2}\right] \\
& =\omega_{n}^{w}+\left(\gamma_{n}^{w}+\beta_{n}^{w}\right) E\left[h_{n-1}\left(\theta_{0}\right)\right] \\
& \leq \omega_{u}^{h} \frac{1-\left(\beta_{u}^{h}+\gamma_{u}^{h}\right)^{n-1}}{1-\left(\beta_{u}^{h}+\gamma_{u}^{h}\right)}+\left(\gamma_{u}^{h}\right)^{n-1} E\left[h_{1}\left(\theta_{0}\right)\right] \\
& \leq \frac{\omega_{u}^{h}}{1-\beta_{u}^{h}-\gamma_{u}^{h}}+E\left[h_{1}\left(\theta_{0}\right)\right]<\infty .
\end{aligned}
$$

Then we can easily show $\sup _{n \in \mathbb{N}} E\left[\sup _{\theta \in \Theta} h_{n}(\theta)\right]<\infty$.

For $(b)$, let $f\left(t, Z_{i, t}\right)=\left(e^{(n-t) \beta_{0, i}}-1\right)\left(Z_{i, t}-Z_{i, n-1}\right)^{2}$. Then, by Itô's lemma, we have

$$
\begin{aligned}
d f\left(t, Z_{i, t}\right)= & \left.-\beta_{0, i} e^{(n-t) \beta_{0, i}}\left(Z_{i, t}-Z_{i, n-1}\right)^{2}+\left(e^{(n-t) \beta_{0, i}}-1\right) \sigma_{i, t}^{2}\right] d t \\
& +2\left(e^{(n-t) \beta_{0, i}}-1\right)\left(Z_{i, t}-Z_{i, n-1}\right) d Z_{i, t}, \\
f\left(n, Z_{i, n}\right)=0= & \int_{n-1}^{n}\left[-\beta_{0, i} e^{(n-t) \beta_{0, i}}\left(Z_{i, t}-Z_{i, n-1}\right)^{2}+\left(e^{(n-t) \beta_{0, i}}-1\right) \sigma_{i, t}^{2}\right] d t+\xi_{i, n} .
\end{aligned}
$$

Consider $(c)$. For any $\delta>0$, there exists a neighborhood $B\left(\theta_{0}\right) \subset \Theta$ such that $\gamma_{0, n}^{w} \leq(1+\delta) \gamma_{n}^{w}$. Using the fact that $x /(1+x) \leq x^{q}$ for all $x \geq 0$ and any $q \in[0,1]$, we have

$$
\begin{aligned}
\frac{h_{n}\left(\theta_{0}\right)}{h_{n}(\theta)} & =\frac{\sum_{k=1}^{n-1}\left(\omega_{0, n-k+1}^{w}+\beta_{0, n-k+1}^{w} Z_{n-k}^{2}\right) \prod_{l=1}^{k-1} \gamma_{0, n-l+1}^{w}+\prod_{k=1}^{n-1} \gamma_{0, n-k+1}^{w} h_{1}\left(\theta_{0}\right)}{\sum_{k=1}^{n-1}\left(\omega_{n-k+1}^{w}+\beta_{n-k+1}^{w} Z_{n-k}^{2}\right) \prod_{l=1}^{k-1} \gamma_{n-l+1}^{w}+\prod_{k=1}^{n-1} \gamma_{n-k+1}^{w} h_{1}(\theta)} \\
& \leq \frac{\sum_{k=1}^{n-1}\left(\omega_{0, n-k+1}^{w}+\beta_{0, n-k+1}^{w} Z_{n-k}^{2}\right) \prod_{l=1}^{k-1} \gamma_{0, n-l+1}^{w}+C}{\omega_{n}^{w}+\sum_{k=2}^{n-1}\left(\omega_{n-k+1}^{w}+\beta_{n-k+1}^{w} Z_{n-k}^{2} \prod_{l=1}^{k-1} \gamma_{n-l+1}^{w}\right)+C}
\end{aligned}
$$




$$
\begin{aligned}
& \leq \sum_{k=1}^{n-1}\left[\frac{\omega_{u}^{h}}{\omega_{l}^{h}}\left(\gamma_{u}^{h}\right)^{k-1}+\frac{\beta_{0, n-k+1}^{w} Z_{n-k}^{2} \prod_{l=1}^{k-1} \gamma_{0, n-l+1}^{w}}{\omega_{l}^{h}+\beta_{n-k+1}^{w} Z_{n-k}^{2} \prod_{l=1}^{k-1} \gamma_{n-l+1}^{w}}\right]+C \\
& =C+\frac{\beta_{l}^{h}}{\beta_{u}^{h}} \sum_{k=1}^{n-1} \frac{\beta_{n-k+1}^{w} Z_{n-k}^{2} \prod_{l=1}^{k-1} \gamma_{n-l+1}^{w}}{\omega_{l}^{h}+\beta_{n-k+1}^{w} Z_{n-k}^{2} \prod_{l=1}^{k-1} \gamma_{n-l+1}^{w}}\left(\frac{\prod_{l=1}^{k-1} \gamma_{0, n-l+1}^{w}}{\prod_{l=1}^{k-1} \gamma_{n-l+1}^{w}}\right) \\
& =C+C \sum_{k=1}^{n-1} \frac{x_{k}}{1+x_{k}} \prod_{l=1}^{k-1} \frac{\gamma_{0, n-l+1}^{w}}{\gamma_{n-l+1}^{w}} \\
& \leq C+C \sum_{k=1}^{n-1} x_{k}^{q} \prod_{l=1}^{k-1} \frac{\gamma_{0, n-l+1}^{w}}{\gamma_{n-l+1}^{w}} \\
& \leq C+C \sum_{k=1}^{n-1}\left(\beta_{u}^{h}\right)^{q} \frac{Z_{n-k}^{2 q}}{\left(\omega_{l}^{h}\right)^{q}}\left(\gamma_{u}^{h}\right)^{q(p-1)}(1+\delta)^{p-1} \\
& =C+C \sum_{k=1}^{n-1}\left(\gamma_{u}^{h}\right)^{q(p-1)}(1+\delta)^{p-1} Z_{n-k}^{2 q} \\
& =C+C \sum_{k=1}^{n-1} \rho^{p-1} Z_{n-k}^{2 q},
\end{aligned}
$$

where $x_{k}=\frac{\beta_{n-k+1}^{w} Z_{n-k}^{2} \prod_{l=1}^{k-1} \gamma_{n-l+1}^{w}}{\omega_{l}^{h}}$. Let $0<\delta<\frac{1-\left(\gamma_{u}^{h}\right)^{q}}{\left(\gamma_{u}^{h}\right)^{q}}$. Then, $(1+\delta)<\frac{1}{\left(\gamma_{u}^{h}\right)^{q}}$ and $\rho=(1+$ $\delta)\left(\gamma_{u}^{h}\right)^{q}<1$. Taking $q \in[0,1]$ such that $E\left(Z_{n-k}^{2 p q}\right)<\infty$, we have

$$
\left\|\sup _{\theta \in B\left(\theta_{0}\right)} \frac{h_{n}\left(\theta_{0}\right)}{h_{n}(\theta)}\right\|_{L_{p}} \leq C+C \sum_{k=1}^{n-1} \rho^{p-1}\left\|Z_{n-k}^{2 q}\right\|_{L_{p}}<\infty .
$$

From $|\rho|<1$, we conclude that

$$
\sup _{n \in \mathbb{N}}\left\|\sup _{\theta \in B\left(\theta_{0}\right)} \frac{h_{n}\left(\theta_{0}\right)}{h_{n}(\theta)}\right\|_{L_{p}}<\infty
$$

For (d), we first examine the first derivatives. For $\omega_{1}, \omega_{2}, \beta_{1}$, and $\beta_{2}$, we can show that

$$
\frac{1}{h_{n}(\theta)} \frac{\partial h_{n}(\theta)}{\partial \theta_{j}} \leq C \quad \text { a.s. for } j=1,2,3,4
$$

because $\sigma_{n}^{2}$ is their linear function.

Consider the case that $\left(\omega_{n}^{w}, \beta_{n}^{w}, \gamma_{n}^{w}\right)=\left(\omega_{21}^{h}, \beta_{21}^{h}, \gamma_{21}^{h}\right)$. Under Assumption 11(a), we can easily 
show that

$$
\left|\frac{\partial \theta_{j}^{h}}{\partial \gamma_{k}}\right| \leq C \quad \text { a.s. for } j=1,2, \ldots, 12 \text { and } k=1,2 \text {. }
$$

The property that $x /(1+x) \leq x^{q}$ for any $q \in[0,1]$ and all $x \geq 0$ gives us

$$
\begin{aligned}
\left|\frac{1}{h_{n}(\theta)} \frac{\partial h_{n}(\theta)}{\partial \gamma_{1}}\right|= & h_{n}(\theta)^{-1}\left|\sum_{k=1}^{n-1} \frac{\partial \omega_{21}^{h}}{\partial \gamma_{1}}\left(\gamma_{21}^{h}\right)^{k-1}+(k-1)\left(\omega_{21}^{h}+\beta_{21}^{h} Z_{n-k}^{2}\right)\left(\gamma_{21}^{h}\right)^{k-2} \frac{\partial \gamma_{21}^{h}}{\partial \gamma_{1}}\right| \\
& +(n-1)\left(\gamma_{21}^{h}\right)^{n-2} h_{1}(\theta) \frac{\partial \gamma_{21}^{h}}{\partial \gamma_{1}} \\
\leq & C\left|\sum_{k=1}^{n-1} \frac{\left(\gamma_{21}^{h}\right)^{k-1}}{\left(\gamma_{21}^{h}\right)^{k-1}\left(\omega_{21}^{h}+\beta_{21}^{h} Z_{n-k}^{2}\right)}\right|+C \mid \sum_{k=1}^{n-1} \frac{(k-1)\left(\gamma_{21}^{h}\right)^{k-2}\left(\omega_{21}^{h}+\beta_{21}^{h} Z_{n-k}^{2}\right)}{\omega_{21}^{h}+\left(\gamma_{21}^{h}\right)^{k-2}\left(\omega_{21}^{h}+\beta_{21}^{h} Z_{n-k}^{2}\right) \mid+C} \\
\leq & C\left|\sum_{k=1}^{n-2} k \rho^{k q}\left(\omega_{u}^{h}+\beta_{u}^{h} Z_{n-k-1}\right)^{q}\right|+C .
\end{aligned}
$$

We can choose $q \in[0,1]$ such that $E\left(\omega_{u}^{h}+\beta_{u}^{h} Z_{n-k-1}\right)^{q p}<\infty$. Then, since $|\rho|<1$,

$$
\sup _{n \in \mathbb{N}}\left\|\frac{1}{h_{n}(\theta)} \frac{\partial h_{n}(\theta)}{\partial \gamma_{1}}\right\|_{L_{p}}<C
$$

Similarly, we can show the bound for the first derivatives of the $h_{n}(\theta)$ and for the second and third derivatives.

Lemma 3. Under Assumption 1(a)-(b) and Assumption 2(a)-(b), we have

$$
\sup _{\theta \in \Theta}\left|\widehat{Q}_{N, M}(\theta)-Q_{N}(\theta)\right|=O_{p}\left(M^{-\frac{1}{4}}\right)+o_{p}(1) .
$$

Proof. Proof. By the triangular inequality, we have

$$
\left|\widehat{Q}_{N, M}(\theta)-Q_{N}(\theta)\right| \leq\left|\widehat{Q}_{N, M}(\theta)-\widetilde{Q}_{N}(\theta)\right|+\left|\widetilde{Q}_{N}(\theta)-Q_{N}(\theta)\right|
$$


Note that $h_{n}(\theta)^{-1}<\infty$ a.s. By Assumption 2(b), we have

$$
E\left[\sup _{\theta \in \Theta}\left|\widehat{Q}_{N, M}(\theta)-\widetilde{Q}_{N}(\theta)\right|\right] \leq C \frac{1}{N} \sum_{n=1}^{N} E\left[\left\|R V_{n}-\int_{n-1}^{n} \sigma_{t}^{2} d t\right\|_{L_{2}}\right] \leq C M^{-\frac{1}{4}}
$$

Accordingly, we have

$$
\sup _{\theta \in \Theta}\left|\widehat{Q}_{N, M}(\theta)-\widetilde{Q}_{N}(\theta)\right|=O_{p}\left(M^{-\frac{1}{4}}\right)
$$

We can easily show that $\widetilde{Q}_{N}(\theta)-Q_{N}(\theta)=\frac{1}{2 N} \sum_{n=1}^{N} \frac{\xi_{n}}{h_{n}(\theta)}$. Because $h_{n}(\theta)$ is adapted to $\mathcal{F}_{n-1}, \frac{\xi_{n}}{h_{n}(\theta)}$ is also martingale difference. Furthermore, $\left|\frac{\xi_{n}}{h_{n}(\theta)}\right|$ is uniformly integrable because $\left|\frac{\xi_{n}}{h_{n}(\theta)}\right| \leq \frac{1}{\omega_{l}^{h}}\left|\xi_{n}\right|$. Thus, $\left|\widetilde{Q}_{N}(\theta)-Q_{N}(\theta)\right| \longrightarrow 0$ in probability (see Theorem 2.22 in Hall and Heyde (2014)).

Let $K_{N}(\theta)=\widetilde{Q}_{N}(\theta)-Q_{N}(\theta)$. By mean-value theorem, there exists $\theta^{*}$ between $\theta$ and $\theta^{\prime}$ satisfying

$$
\begin{aligned}
\left|K_{N}(\theta)-K_{N}\left(\theta^{\prime}\right)\right| & =\left|\frac{1}{2 N} \sum_{n=1}^{N} \frac{\xi_{n}}{h_{n}^{2}\left(\theta^{*}\right)} \frac{\partial h_{n}\left(\theta^{*}\right)}{\partial \theta}\left(\theta-\theta^{\prime}\right)\right| \\
& \leq \frac{1}{2 N} \sum_{n=1}^{N}\left\|\sup _{\theta^{*} \in \Theta} \frac{\xi_{n}}{h_{n}^{2}\left(\theta^{*}\right)} \frac{\partial h_{n}\left(\theta^{*}\right)}{\partial \theta}\right\|_{\text {max }}\left\|\left(\theta-\theta^{\prime}\right)\right\|_{\text {max }} .
\end{aligned}
$$

By Lemma 2(d), $\left\|\frac{\partial h_{n}\left(\theta^{*}\right)}{\partial \theta_{k}} \frac{1}{h_{n}\left(\theta^{*}\right)}\right\|_{L_{2}} \leq C$ for every $k \in\{1,2,3,4,5,6\}$. Therefore, we have

$$
\left\|\sup _{\theta^{*} \in \Theta} \frac{\xi_{n}}{h_{n}^{2}\left(\theta^{*}\right)} \frac{\partial h_{n}\left(\theta^{*}\right)}{\partial \theta}\right\|_{L_{1}} \leq C\left\|\xi_{n}\right\|_{L_{1}} \leq C<\infty
$$

As a result, $K_{N}(\theta)$ satisfies the weak Lipschitz condtion and uniformly converges to zero by Theorem 3 in Andrews (1992).

Proof. Proof of Theorem 2 First, let us show the existence of the unique maximizer of $Q_{N}(\theta)$. From the definition of $Q_{N}(\theta)$, it is obvious that

$$
\max _{\theta \in \Theta} Q_{N}(\theta) \leq-\frac{1}{2 N} \sum_{n=1}^{N} \min _{\theta_{n} \in \Theta}\left[\log \left(h_{n}\left(\theta_{n}\right)\right)+\frac{h_{n}\left(\theta_{0}\right)}{h_{n}\left(\theta_{n}\right)}\right]
$$


If $\theta_{0, n}$ is the minimizer of the $n$th summand on right hand side, $\theta_{0, n}$ must satisfy $h_{n}\left(\theta_{0, n}\right)=h_{n}\left(\theta_{0}\right)$ for every $n \in \mathbb{N}$. Therefore, if there exists $\theta^{*} \in \Theta$ such that $h_{n}\left(\theta^{*}\right)=h_{n}\left(\theta_{0}\right)$ for every $n \in \mathbb{N}, \theta^{*}$ would be the maximizer. In this manner, $\theta_{0}$ is one of the candidates of $\theta^{*}$. We then show that $\theta^{*}=\theta_{0}$ a.s.

Under the SG-Itô framework, we have

$$
\begin{aligned}
h_{n}(\theta)= & s_{11, n}\left(\omega_{11}^{h}+\gamma_{11}^{h} h_{n-1}(\theta)+\beta_{11}^{h} Z_{n-1}^{2}\right)+s_{12, n}\left(\omega_{12}^{h}+\gamma_{12}^{h} h_{n-1}(\theta)+\beta_{12}^{h} Z_{n-1}^{2}\right) \\
& +s_{21, n}\left(\omega_{21}^{h}+\gamma_{21}^{h} h_{n-1}(\theta)+\beta_{21}^{h} Z_{n-1}^{2}\right)+s_{22, n}\left(\omega_{22}^{h}+\gamma_{22}^{h} h_{n-1}(\theta)+\beta_{22}^{h} Z_{n-1}^{2}\right) .
\end{aligned}
$$

Then $\theta^{*}$ and $\theta_{0}$ satisfy $A P=0$ a.s., where

$$
\begin{aligned}
& \theta^{*}=\left(\omega_{1}^{*}, \omega_{2}^{*}, \gamma_{1}^{*}, \gamma_{2}^{*}, \beta_{1}^{*}, \beta_{2}^{*}\right) \\
& A=\left(\begin{array}{ccccccccc}
s_{11,1} & \cdots & s_{22,1} & s_{11,1} h_{1}\left(\theta_{0}\right) & \cdots & s_{22,1} h_{1}\left(\theta_{0}\right) & s_{11,1} Z_{1}^{2} & \cdots & s_{22,1} Z_{1}^{2} \\
s_{11,2} & \cdots & s_{22,2} & s_{11,2} h_{2}\left(\theta_{0}\right) & \cdots & s_{22,2} h_{2}\left(\theta_{0}\right) & s_{11,2} Z_{2}^{2} & \cdots & s_{22,2} Z_{2}^{2} \\
\vdots & & \vdots & \vdots & & \vdots & \vdots & & \vdots \\
s_{11, n} & \cdots & s_{22, n} & s_{11, n} h_{n}(\theta) & \cdots & s_{22, n} h_{n}(\theta) & s_{11, n} Z_{n}^{2} & \cdots & s_{22, n} Z_{n}^{2}
\end{array}\right), \\
& P^{T}=\left(\omega_{11}^{h *}-\omega_{0,11}^{h} \omega_{12}^{h *}-\omega_{0,12}^{h} \omega_{21}^{h *}-\omega_{0,21}^{h} \omega_{22}^{h *}-\omega_{0,22}^{h} \gamma_{11}^{h *}-\gamma_{0,11}^{h} \gamma_{12}^{h *}-\gamma_{0,12}^{h}\right. \\
& \left.\gamma_{21}^{h *}-\gamma_{0,21}^{h} \gamma_{22}^{h *}-\gamma_{0,22}^{h} \beta_{11}^{h *}-\beta_{0,11}^{h} \beta_{12}^{h *}-\beta_{0,12}^{h} \beta_{21}^{h *}-\beta_{0,21}^{h} \beta_{22}^{h *}-\beta_{0,22}^{h}\right) \text {. }
\end{aligned}
$$

Note that $A$ is of full rank because $Z_{n}^{2}$ is nondegenerated and $s_{n}=0$ or 1 . Then $A^{T} A$ is invertable and $P=0$ a.s. That is, we have

$$
\omega_{11}^{h *}=\omega_{0,11}^{h}, \omega_{22}^{h *}=\omega_{0,22}^{h}, \gamma_{11}^{h *}=\gamma_{0,11}^{h}, \gamma_{22}^{h *}=\gamma_{0,22}^{h}, \beta_{11}^{h *}=\beta_{0,11}^{h}, \beta_{22}^{h *}=\beta_{0,22}^{h} \quad \text { a.s. }
$$

which implies $\theta^{*}=\theta_{0}$ a.s. This also implies that there is a unique maximizer of $Q_{N}(\theta)$ because $\omega_{11}^{h}\left(\omega_{22}^{h}\right)$ and $\beta_{11}^{h}\left(\beta_{22}^{h}\right)$ are strictly increasing function of $\beta_{1}\left(\beta_{2}\right)$. Then, for any $\epsilon>0$, there is a 
constant $\nu$ such that

$$
Q_{N}\left(\theta_{0}\right)-\max _{\theta \in \Theta:\left\|\theta-\theta_{0}\right\|_{\max }>\epsilon} Q_{N}(\theta)>\nu \quad \text { a.s. }
$$

Now, the theorem is the result of Theorem 1 in Xiu (2010).

\section{A.4.3 Proof of Theorem 3}

Lemma 4. We have following properties under Assumption 1(a), Assumption 2(c), and Lemma 2(d):

(a) There exists a neighborhood $B\left(\theta_{0}\right)$ of $\theta_{0}$ such that $\sup _{n \in \mathbb{N}}\left\|\sup _{\theta \in B\left(\theta_{0}\right)} \frac{\partial^{3} \widehat{q}_{N, M}(\theta)}{\partial \theta_{j} \partial \theta_{k} \partial \theta_{l}}\right\|_{L_{1}}<\infty$ for any $j, k, l \in\{1,2,3,4,5,6\}$, where $\theta=\left(\theta_{1}, \theta_{2}, \theta_{3}, \theta_{4}, \theta_{5}, \theta_{6}\right)=\left(\omega_{1}, \omega_{2}, \gamma_{1}, \gamma_{2}, \beta_{1}, \beta_{2}\right)$.

(b) $-\nabla S_{N}\left(\theta_{0}\right)$ is a positive definite matrix for $N \geq 3$

Proof. Proof. Consider (a). By Assumption 2(c), we have

$$
E\left[R V_{n} \mid \mathcal{F}_{n-1}\right] \leq C E\left[\int_{n-1}^{n} \sigma_{t}^{2} d t \mid \mathcal{F}_{n-1}\right]+C=C h_{n}\left(\theta_{0}\right)+C \text { a.s. }
$$

Then, by Lemma 2(c) and (d), we have

$$
\begin{aligned}
& E\left[\sup _{\theta \in B\left(\theta_{0}\right)}\left|\frac{R V_{n}}{h_{n}(\theta)} \frac{\partial^{3} h_{n}(\theta)}{\partial \theta_{j} \partial \theta_{k} \partial \theta_{l}}\right|\right] \\
& \quad \leq C E\left[\sup _{\theta \in B\left(\theta_{0}\right)} \frac{h_{n}\left(\theta_{0}\right)}{h_{n}(\theta)}\left|\frac{1}{h_{n}(\theta)} \frac{\partial^{3} h_{n}(\theta)}{\partial \theta_{j} \partial \theta_{k} \partial \theta_{l}}\right|\right]+C \\
& \quad \leq C\left\|\sup _{\theta \in B\left(\theta_{0}\right)} \frac{h_{n}\left(\theta_{0}\right)}{h_{n}(\theta)}\right\|\left\|_{L_{2}}\right\| \sup _{\theta \in B\left(\theta_{0}\right)}\left|\frac{1}{h_{n}(\theta)} \frac{\partial^{3} h_{n}(\theta)}{\partial \theta_{j} \partial \theta_{k} \partial \theta_{l}}\right| \|_{L_{2}}+C \leq C<\infty .
\end{aligned}
$$

We can similarly bound remaining terms.

Consider (b). Let

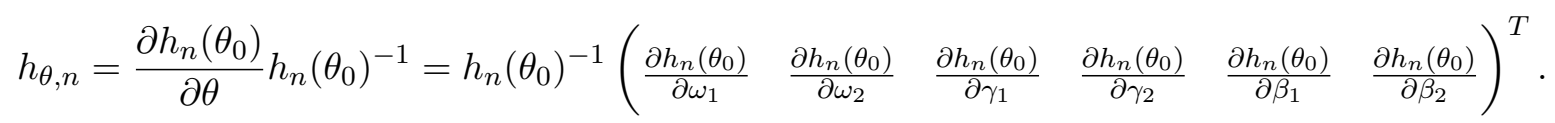


Then we can express $-\nabla S_{N}\left(\theta_{0}\right)=\frac{1}{2 N} \sum_{n=1}^{N} h_{\theta, n} h_{\theta, n}^{T}$. Suppose that $-\nabla S_{N}\left(\theta_{0}\right)$ is not a positive definite matrix. This implies the existence of $\lambda \neq 0$ which satisfies $\frac{1}{2 N} \sum_{n=1}^{N} \lambda^{T} h_{\theta, n} h_{\theta, n}^{T} \lambda=0$, implying that $h_{\theta, n}^{T} \lambda=0$ for all $n=1, \ldots, N$. Define

$$
\begin{aligned}
J= & \left(h_{\theta, 1} h_{\theta, 2} \ldots h_{\theta, n}\right) \\
& =\left(\begin{array}{llll}
\frac{\partial h_{1}\left(\theta_{0}\right)}{\partial \omega_{1}} & \ldots & \ldots & \frac{\partial \omega_{n}^{w}}{\partial \omega_{1}}+\frac{\partial \gamma_{n}^{w}}{\partial \omega_{1}} h_{n-1}\left(\theta_{0}\right)+\gamma_{n}^{w} \frac{\partial h_{n-1}\left(\theta_{0}\right)}{\partial \omega_{1}}+\frac{\partial \beta_{n}^{w}}{\partial \omega_{1}} Z_{n-1}^{2} \\
\frac{\partial h_{1}\left(\theta_{0}\right)}{\partial \omega_{2}} & \ldots & \ldots & \frac{\partial \omega_{n}^{w}}{\partial \omega_{2}}+\frac{\partial \gamma_{n}^{w}}{\partial \omega_{2}} h_{n-1}\left(\theta_{0}\right)+\gamma_{n}^{w} \frac{\partial h_{n-1}\left(\theta_{0}\right)}{\partial \omega_{2}}+\frac{\partial \beta_{n}^{w}}{\partial \omega_{2}} Z_{n-1}^{2} \\
\frac{\partial h_{1}\left(\theta_{0}\right)}{\partial \gamma_{1}} & \ldots & \ldots & \frac{\partial \omega_{n}^{w}}{\partial \gamma_{1}}+\frac{\partial \gamma_{n}^{w}}{\partial \gamma_{1}} h_{n-1}\left(\theta_{0}\right)+\gamma_{n}^{w} \frac{\partial h_{n-1}\left(\theta_{0}\right)}{\partial \gamma_{1}}+\frac{\partial \beta_{n}^{w}}{\partial \gamma_{1}} Z_{n-1}^{2} \\
\frac{\partial h_{1}\left(\theta_{0}\right)}{\partial \gamma_{2}} & \ldots & \ldots & \frac{\partial \omega_{n}^{w}}{\partial \gamma_{2}}+\frac{\partial \gamma_{n}^{w}}{\partial \gamma_{2}} h_{n-1}\left(\theta_{0}\right)+\gamma_{n}^{w} \frac{\partial h_{n-1}\left(\theta_{0}\right)}{\partial \gamma_{2}}+\frac{\partial \beta_{n}^{w}}{\partial \gamma_{2}} Z_{n-1}^{2} \\
\frac{\partial h_{1}\left(\theta_{0}\right)}{\partial \beta_{1}} & \ldots & \ldots & \frac{\partial \omega_{n}^{w}}{\partial \beta_{1}}+\frac{\partial \gamma_{n}^{w}}{\partial \beta_{1}} h_{n-1}\left(\theta_{0}\right)+\gamma_{n}^{w} \frac{\partial h_{n-1}\left(\theta_{0}\right)}{\partial \beta_{1}}+\frac{\partial \beta_{n}^{w}}{\partial \beta_{1}} Z_{n-1}^{2} \\
\frac{\partial h_{1}\left(\theta_{0}\right)}{\partial \beta_{2}} & \ldots & \ldots & \frac{\partial \omega_{n}^{w}}{\partial \beta_{2}}+\frac{\partial \gamma_{n}^{w}}{\partial \beta_{2}} h_{n-1}\left(\theta_{0}\right)+\gamma_{n}^{w} \frac{\partial h_{n-1}\left(\theta_{0}\right)}{\partial \beta_{2}}+\frac{\partial \beta_{n}^{w}}{\partial \beta_{2}} Z_{n-1}^{2}
\end{array}\right),
\end{aligned}
$$

where

$$
\begin{aligned}
& \frac{\partial \omega_{n}^{w}}{\partial \theta_{k}}=s_{11, n} \frac{\partial \omega_{11}^{h}}{\partial \theta_{k}}+s_{12, n} \frac{\partial \omega_{12}^{h}}{\partial \theta_{k}}+s_{21, n} \frac{\partial \omega_{21}^{h}}{\partial \theta_{k}}+s_{22, n} \frac{\partial \omega_{22}^{h}}{\partial \theta_{k}} \\
& \frac{\partial \gamma_{n}^{w}}{\partial \theta_{k}}=s_{11, n} \frac{\partial \gamma_{11}^{h}}{\partial \theta_{k}}+s_{12, n} \frac{\partial \gamma_{12}^{h}}{\partial \theta_{k}}+s_{21, n} \frac{\partial \gamma_{21}^{h}}{\partial \theta_{k}}+s_{22, n} \frac{\partial \gamma_{22}^{h}}{\partial \theta_{k}} \\
& \frac{\partial \beta_{n}^{w}}{\partial \theta_{k}}=s_{11, n} \frac{\partial \beta_{11}^{h}}{\partial \theta_{k}}+s_{12, n} \frac{\partial \beta_{12}^{h}}{\partial \theta_{k}}+s_{21, n} \frac{\partial \beta_{21}^{h}}{\partial \theta_{k}}+s_{22, n} \frac{\partial \beta_{22}^{h}}{\partial \theta_{k}}
\end{aligned}
$$

for any $k \in\{1,2,3,4,5,6\}$ and $\theta=\left(\theta_{1}, \theta_{2}, \theta_{3}, \theta_{4}, \theta_{5}, \theta_{6}\right)=\left(\omega_{1}, \omega_{2}, \gamma_{1}, \gamma_{2}, \beta_{1}, \beta_{2}\right)$. Since $Z_{i}$ 's are nondegenerated, $J$ is of full rank almost surely. Therefore, $J^{T} \lambda=0$ a.s. implies $\lambda=0$ a.s., which is contradiction.

Proof. Proof of Theorem 3. By mean-value theorem, there exists $\theta^{*}$ between $\widehat{\theta}$ and $\theta_{0}$ such that $\widehat{S}_{N, M}(\widehat{\theta})-\widehat{S}_{N, M}\left(\theta_{0}\right)=-\widehat{S}_{N, M}\left(\theta_{0}\right)=\nabla \widehat{S}_{N, M}\left(\theta^{*}\right)\left(\widehat{\theta}-\theta_{0}\right)$. If $-\nabla \widehat{S}_{N, M}\left(\theta^{*}\right) \stackrel{p}{\longrightarrow}-\nabla S_{N}\left(\theta_{0}\right)$, which is a positive definite matrix, convergence rates of $\left|\widehat{S}_{N, M}\left(\theta_{0}\right)\right|$ and $\left|\widehat{\theta}-\theta_{0}\right|$ are equivalent. Therefore, proof of Theorem 3 is equivalent to show (a) $\widehat{S}_{N, M}\left(\theta_{0}\right)=O_{p}\left(M^{-\frac{1}{4}}+N^{-\frac{1}{2}}\right)$ and (b) $\left\|\nabla \widehat{S}_{N, M}\left(\theta^{*}\right)-\nabla S_{N}\left(\theta_{0}\right)\right\|_{\max }=$ 
$o_{p}(1)$. Consider (a). By Assumption Lemma 2(d) and Assumption 2(b), we have

$$
\begin{aligned}
\left\|\widehat{S}_{N, M}\left(\theta_{0}\right)-\widetilde{S}_{N}\left(\theta_{0}\right)\right\|_{L_{1}} & =\frac{1}{2 N}\left\|\sum_{n=1}^{N} \frac{\partial h_{n}\left(\theta_{0}\right)}{\partial \theta} \frac{1}{h_{n}\left(\theta_{0}\right)^{2}}\left(R V_{n}-\int_{n-1}^{n} \sigma_{t}^{2} d t\right)\right\|_{L_{1}} \\
& \leq \frac{1}{2 N} \sum_{n=1}^{N}\left\|\frac{\partial h_{n}\left(\theta_{0}\right)}{\partial \theta} \frac{1}{h_{n}\left(\theta_{0}\right)^{2}}\right\|_{L_{2}}\left\|R V_{n}-\int_{n-1}^{n} \sigma_{t}^{2} d t\right\|_{L_{2}} \\
& \leq C M^{-\frac{1}{4}} .
\end{aligned}
$$

Then we have

$$
\begin{aligned}
\widehat{S}_{N, M}\left(\theta_{0}\right)-S_{N}\left(\theta_{0}\right)=\widehat{S}_{N, M}\left(\theta_{0}\right) & =-\frac{1}{2 N} \sum_{n=1}^{N} \frac{\partial h_{n}\left(\theta_{0}\right)}{\partial \theta} \frac{1}{h_{n}\left(\theta_{0}\right)} \frac{\left(R V_{n}-\int_{n-1}^{n} \sigma_{t}^{2} d t\right)+\xi_{n}}{h_{n}\left(\theta_{0}\right)} \\
& =-\frac{1}{2 N} \sum_{n=1}^{N} \frac{\partial h_{n}\left(\theta_{0}\right)}{\partial \theta} \frac{1}{h_{n}\left(\theta_{0}\right)} \frac{\xi_{n}}{h_{n}\left(\theta_{0}\right)}+O_{p}\left(M^{-\frac{1}{4}}\right) .
\end{aligned}
$$

By Itô's lemma and Itô's isometry, for $j \in\{1,2,3,4,5,6\}$, we have

$$
\begin{aligned}
& E\left[\left(\frac{1}{2 N} \sum_{n=1}^{N} \frac{\partial h_{n}\left(\theta_{0}\right)}{\partial \theta_{j}} \frac{1}{h_{n}\left(\theta_{0}\right)} \frac{\xi_{n}}{h_{n}\left(\theta_{0}\right)}\right)^{2}\right] \\
& =\frac{1}{4 N^{2}} E\left[\sum_{n=1}^{N}\left(\frac{\partial h_{n}\left(\theta_{0}\right)}{\partial \theta_{j}}\right)^{2}\left(\frac{1}{h_{n}\left(\theta_{0}\right)}\right)^{2}\left(\frac{\xi_{n}}{h_{n}\left(\theta_{0}\right)}\right)^{2}\right] \\
& =\frac{1}{4 N^{2}} E\left[\sum_{n=1}^{N}\left(\frac{\partial h_{n}\left(\theta_{0}\right)}{\partial \theta_{j}}\right)^{2}\left(\frac{1}{h_{n}\left(\theta_{0}\right)}\right)^{2} \frac{E\left[\xi_{n}^{2} \mid \mathcal{F}_{n-1}\right]}{h_{n}\left(\theta_{0}\right)^{2}}\right] \\
& \leq C \frac{1}{N^{2}} E\left[\sum_{n=1}^{N}\left(\frac{\partial h_{n}\left(\theta_{0}\right)}{\partial \theta_{j}}\right)^{2}\left(\frac{1}{h_{n}\left(\theta_{0}\right)}\right)^{2} \frac{E\left[Z_{n}^{4} \mid \mathcal{F}_{n-1}\right]}{h_{n}\left(\theta_{0}\right)^{2}}\right] \\
& \leq C \frac{1}{N^{2}} \sum_{n=1}^{N}\left\|\left(\frac{\partial h_{n}\left(\theta_{0}\right)}{\partial \theta_{j}}\right)^{2}\left(\frac{1}{h_{n}\left(\theta_{0}\right)}\right)^{2}\right\|_{L_{2}}\left\|\frac{E\left[Z_{n}^{4} \mid \mathcal{F}_{n-1}\right]}{h_{n}\left(\theta_{0}\right)^{2}}\right\|_{L_{2}} \\
& =O_{p}\left(N^{-1}\right)
\end{aligned}
$$

where the last equality is hold by Lemma 2(d) and Assumption 1(c). Therefore, the statement of (a) is proved. 
Consider (b). By the triangular inequality, we have

$$
\begin{aligned}
\left\|\nabla \widehat{S}_{N, M}\left(\theta^{*}\right)-\nabla S_{N}\left(\theta_{0}\right)\right\|_{\max } \leq & \left\|\nabla \widehat{S}_{N, M}\left(\theta^{*}\right)-\nabla \widehat{S}_{N, M}\left(\theta_{0}\right)\right\|_{\max } \\
& +\left\|\nabla \widehat{S}_{N, M}\left(\theta_{0}\right)-\nabla S_{N}\left(\theta_{0}\right)\right\|_{\max }
\end{aligned}
$$

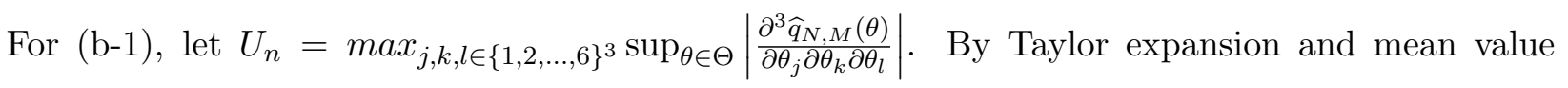
theorem, following inequality is satisfied for $\theta^{* *}$ between $\theta_{0}$ and $\theta^{*}$ :

$$
\begin{aligned}
\left\|\nabla \widehat{S}_{N, M}\left(\theta^{*}\right)-\nabla \widehat{S}_{N, M}\left(\theta_{0}\right)\right\|_{\max } & \leq \frac{1}{2 N} \sum_{n=1}^{N}\left\|\frac{\partial^{3} \widehat{q}_{N, M}\left(\theta^{* *}\right)}{\partial \theta_{j} \partial \theta_{k} \partial \theta_{l}}\right\|_{\max }\left\|\theta^{*}-\theta_{0}\right\|_{\max } \\
& \leq C \frac{1}{2 N} \sum_{n=1}^{N} U_{n}\left\|\theta^{*}-\theta_{0}\right\|_{\max }=o_{p}(1)
\end{aligned}
$$

where the last line is due to Theorem 2 and Lemma 4. For (b-2), by Lemma 2(d) and Assumption 2(b), we have

$$
\begin{aligned}
& \left\|\nabla \widehat{S}_{N, M}\left(\theta_{0}\right)-\nabla \widetilde{S}_{N}\left(\theta_{0}\right)\right\|_{\max } \\
& =\left\|\frac{1}{2 N} \sum_{n=1}^{N}\left[\frac{2}{h_{n}(\theta)^{3}} \frac{\partial h_{n}\left(\theta_{0}\right)}{\partial \theta} \frac{\partial h_{n}\left(\theta_{0}\right)}{\partial \theta^{T}}-\frac{1}{h_{n}(\theta)^{2}} \frac{\partial^{2} h_{n}\left(\theta_{0}\right)}{\partial \theta \partial \theta^{T}}\right]\left(R V_{n}-\int_{n-1}^{n} \sigma_{t}^{2} d t\right)\right\|_{\max } \\
& =O_{p}\left(M^{-\frac{1}{4}}\right) .
\end{aligned}
$$

Note that we have

$$
\begin{aligned}
\eta_{n}= & \nabla \widehat{S}_{N}\left(\theta_{0}\right)-\nabla S_{N}\left(\theta_{0}\right) \\
= & \frac{1}{2 N} \sum_{n=1}^{N} \frac{\partial^{2} h_{n}\left(\theta_{0}\right)}{\partial \theta \partial \theta^{T}} h_{n}\left(\theta_{0}\right)^{-1}\left(\frac{\int_{n-1}^{n} \sigma_{t}^{2} d t-h_{n}\left(\theta_{0}\right)}{h_{n}\left(\theta_{0}\right)}\right) \\
& -2 \frac{\partial h_{n}\left(\theta_{0}\right)}{\partial \theta} \frac{\partial h_{n}\left(\theta_{0}\right)}{\partial \theta^{T}} h_{n}\left(\theta_{0}\right)^{-2} \frac{\int_{n-1}^{n} \sigma_{t}^{2} d t-h_{n}\left(\theta_{0}\right)}{h_{n}\left(\theta_{0}\right)} \\
= & \frac{1}{2 N} \sum_{n=1}^{N} \frac{\xi_{n}}{h_{n}\left(\theta_{0}\right)}\left[\frac{\partial^{2} h_{n}\left(\theta_{0}\right)}{\partial \theta \partial \theta^{T}} h_{n}\left(\theta_{0}\right)^{-1}-2 \frac{\partial h_{n}\left(\theta_{0}\right)}{\partial \theta} \frac{\partial h_{n}\left(\theta_{0}\right)}{\partial \theta^{T}} h_{n}\left(\theta_{0}\right)^{-2}\right] .
\end{aligned}
$$


We can easily show that $\left\|\eta_{n}^{2}\right\|_{\max } \leq C \frac{1}{N^{2}} \sum_{n=1}^{N}\left\|\frac{E\left[Z_{n}^{4} \mid \mathcal{F}_{n-1}\right]}{h_{n}\left(\theta_{0}\right)^{2}}\right\|_{\max }=O_{p}\left(N^{-1}\right)$. As a result, we have

$$
\begin{aligned}
\nabla \widehat{S}_{N, M}\left(\theta_{0}\right) & =\nabla \widetilde{S}_{N}\left(\theta_{0}\right)+O_{p}\left(M^{-\frac{1}{4}}\right) \\
& =\nabla S_{N}\left(\theta_{0}\right)+\eta_{n}+O_{p}\left(M^{-\frac{1}{4}}\right) \\
& =\nabla S_{N}\left(\theta_{0}\right)+O_{p}\left(N^{-\frac{1}{2}}\right)+O_{p}\left(M^{-\frac{1}{4}}\right) .
\end{aligned}
$$

\section{A.4.4 Proof of Theorem 4}

Proof. Proof of Theorem 4. For any $\lambda \in \mathbb{R}^{6}$, let $v_{n}=\frac{\partial h_{n}\left(\theta_{0}\right)}{\partial \theta} h_{n}\left(\theta_{0}\right)^{-2} \xi_{n}$ and $\kappa_{n}=\lambda^{T} v_{n}$. Since $\kappa_{n}$ is martingale difference, $E\left(\kappa_{n}^{2}\right)<\infty$. Also, $\kappa_{n}$ is stationary and ergodic by Assumption 1(d). Let $\frac{1}{\sqrt{N}} \sum_{n=1}^{N} \kappa_{n}^{2} \stackrel{p}{\longrightarrow} \kappa$. By martingale CLT, $\frac{1}{\sqrt{N}} \kappa^{-\frac{1}{2}} \sum_{n=1}^{N} \kappa_{n} \stackrel{d}{\longrightarrow} N(0,1)$. Let $\frac{1}{4 N} \sum_{n=1}^{N} v_{n} v_{n}^{T} \stackrel{p}{\longrightarrow} V$. Then, by Cramer-Wold device, we have

$$
-\sqrt{N} V^{-\frac{1}{2}} \widetilde{S}_{N}\left(\theta_{0}\right)=\frac{\sqrt{N}}{2 N} V^{-\frac{1}{2}} \sum_{n=1}^{N} v_{n} \stackrel{d}{\longrightarrow} N\left(0, I_{6}\right),
$$

where $I_{k}$ denotes $\mathrm{k}$ by k identity matrix. Furthermore, let

$$
-\nabla S_{N}\left(\theta_{0}\right)=\frac{1}{2 N} \sum_{n=1}^{N}\left[\frac{\partial h_{n}\left(\theta_{0}\right)}{\partial \theta} \frac{\partial h_{n}\left(\theta_{0}\right)}{\partial \theta^{T}} h_{n}\left(\theta_{0}\right)^{-2}\right] \stackrel{p}{\longrightarrow} W
$$

for positive definite matrix $W$. By mean-value theorem, there exists $\theta^{*}$ between $\widehat{\theta}$ and $\theta_{0}$ which satisfies

$$
\widehat{S}_{N, M}(\widehat{\theta})-\widehat{S}_{N, M}\left(\theta_{0}\right)=-\widehat{S}_{N, M}\left(\theta_{0}\right)=\nabla \widehat{S}_{N, M}\left(\theta^{*}\right)\left(\widehat{\theta}-\theta_{0}\right)
$$


Then we have

$$
\begin{aligned}
\sqrt{N}\left(\widehat{\theta}-\theta_{0}\right) & =-\sqrt{N} \nabla \widehat{S}_{N, M}\left(\theta^{*}\right)^{-1} \widehat{S}_{N, M}\left(\theta_{0}\right) \\
& =\sqrt{N}\left(W^{-1}+o_{p}(1)\right)\left(\widetilde{S}_{N}\left(\theta_{0}\right)+O_{p}\left(M^{-\frac{1}{4}}\right)\right) \\
& =\sqrt{N} \widetilde{S}_{N}\left(\theta_{0}\right) W^{-1}+O_{p}\left(N^{\frac{1}{2}} M^{-\frac{1}{4}}\right)+o_{p}(1) .
\end{aligned}
$$

Thus, we can conclude that

$$
\left(W^{-1} V W^{-1}\right)^{-\frac{1}{2}} \sqrt{N}\left(\widehat{\theta}-\theta_{0}\right) \stackrel{d}{\longrightarrow} N\left(0, I_{6}\right)
$$

\section{A.4.5 Proof of Proposition 2}

Proof. Proof of Proposition 2. We first consider $\widehat{V}$. Let

$$
\begin{aligned}
& I(\theta)=\frac{1}{4 N} \sum_{n=1}^{N}\left[\left.\frac{\partial h_{n}(\theta)}{\partial \theta}\left[\frac{\partial h_{n}(\theta)}{\partial \theta}\right]^{T}\right|_{\theta=\theta} h_{n}(\theta)^{-4}\left(R V_{n}-h_{n}(\theta)\right)^{2}\right]=\frac{1}{4 N} \sum_{n=1}^{N} \iota_{n}(\theta), \\
& \tilde{I}(\theta)=\frac{1}{4 N} \sum_{n=1}^{N}\left[\left.\frac{\partial h_{n}(\theta)}{\partial \theta}\left[\frac{\partial h_{n}(\theta)}{\partial \theta}\right]^{T}\right|_{\theta=\theta} h_{n}(\theta)^{-4} \xi_{n}^{2}\right]=\frac{1}{4 N} \sum_{n=1}^{N} \tilde{\iota}_{n}(\theta) .
\end{aligned}
$$

Then, $\widehat{V}=I(\widehat{\theta})$ and we have

$$
\|I(\widehat{\theta})-V\|_{\max } \leq\left\|I(\widehat{\theta})-I\left(\theta_{0}\right)\right\|_{\max }+\left\|I\left(\theta_{0}\right)-V\right\|_{\max }
$$

First, we show that the convergence of $I(\widehat{\theta})$ to $I\left(\theta_{0}\right)$ is equivalent to that of $\widehat{\theta}$ to $\theta_{0}$. Similar to the proof of Lemma 4(a), under Lemma 2(d) and Assumption 2(c), we can show $U_{n}^{\prime}=$ $\max _{j} \sup _{\theta \in B\left(\theta_{0}\right)}\left|\frac{\partial \iota_{n}(\theta)}{\partial \theta_{j}}\right|=O_{p}(1)$ for $j \in\{1,2,3,4,5,6\}$. For large $N$ and $M$, by mean value theorem and Taylor expansion, we have

$$
\left\|I(\widehat{\theta})-I\left(\theta_{0}\right)\right\|_{\max } \leq \frac{C}{4 N} \sum_{n=1}^{N} U_{n}^{\prime}\left\|\widehat{\theta}-\theta_{0}\right\|_{\max }
$$


Since Theorem 3 shows that $\widehat{\theta}$ converges to $\theta_{0}$ with the convergence rate $N^{-1 / 2}+M^{-1 / 4}$, we have

$$
\left\|I(\widehat{\theta})-I\left(\theta_{0}\right)\right\|_{\max }=O_{p}\left(N^{-1 / 2}+M^{-1 / 4}\right) .
$$

For the second term of right hand side of Equation (A.6), we have

$$
\left\|I\left(\theta_{0}\right)-V\right\|_{\max } \leq\left\|I\left(\theta_{0}\right)-\tilde{I}\left(\theta_{0}\right)\right\|_{\max }+\left\|\tilde{I}\left(\theta_{0}\right)-V\right\|_{\max }
$$

We consider the first term of right hand side of Equation A.7). For $j \in\{1,2,3,4,5,6\}$, we have

$$
\begin{aligned}
& \frac{1}{4 N} \sum_{n=1}^{N} E\left[\frac{1}{4 N} \sum_{n=1}^{N}\left(\frac{\partial h_{n}\left(\theta_{0}\right)}{\partial \theta_{j}}\right)^{2} h_{n}\left(\theta_{0}\right)^{-4}\left\{\left(R V-h_{n}\left(\theta_{0}\right)\right)^{2}-\xi^{2}\right\}\right] \\
& \leq \frac{C}{4 N} \sum_{n=1}^{N}\left\|h_{n}\left(\theta_{0}\right)^{-1}\left\{\left(R V-h_{n}\left(\theta_{0}\right)\right)^{2}-\left(\int_{n-1}^{n} \sigma_{t}^{2} d t-h_{n}\left(\theta_{0}\right)\right)^{2}\right\}\right\|_{L_{2}} \\
& \leq \frac{C}{4 N} \sum_{n=1}^{N}\left\|R V-\int_{n-1}^{n} \sigma_{t}^{2} d t\right\|_{L_{4}}\left\|\frac{R V-\int_{n-1}^{n} \sigma_{t}^{2} d t+2 \xi}{h_{n}\left(\theta_{0}\right)}\right\|_{L_{4}} \\
& \leq C M^{-\frac{1}{2}}
\end{aligned}
$$

where the first inequality is hold by Lemma 2(d) and the last inequality is hold by Assumption 3 . Consequently, we have $\left\|I\left(\theta_{0}\right)-\tilde{I}\left(\theta_{0}\right)\right\|_{\max }=O_{p}\left(M^{-\frac{1}{2}}\right)$ and $\left\|\tilde{I}\left(\theta_{0}\right)-V\right\|_{\max }=o_{p}(1)$. In conclusion, we have

$$
\|I(\widehat{\theta})-V\|_{\max }=o_{p}(1)+O_{p}\left(N^{-1 / 2}+M^{-1 / 4}\right)
$$

Similarly, we can show that $\|\widehat{W}-W\|_{\max }=o_{p}(1)+O_{p}\left(N^{-1 / 2}+M^{-1 / 4}\right)$ as well.

\section{A.4.6 Proof of Theorem 5}

Proof. Proof of Theorem 5. By multiplying both sides of Equation A.5 by $R$, we obtain

$$
\sqrt{N} R\left(\widehat{\theta}-\theta_{0}\right)=-R \sqrt{N} \widetilde{S}_{N}\left(\theta_{0}\right) W^{-1}+O_{p}\left(N^{\frac{1}{2}} M^{-\frac{1}{4}}\right)+o_{p}(1) .
$$


By Theorem 4, we have

$$
\left[R W^{-1} V W^{-1} R^{T}\right]^{\frac{1}{2}} \sqrt{N}(R \widehat{\theta}-r) \stackrel{d}{\longrightarrow} N\left(0, I_{v}\right)
$$

Then, by continuous mapping theorem, we have

$$
N(R \widehat{\theta}-r)^{T}\left(R W^{-1} V W^{-1} R^{T}\right)^{-1}(R \widehat{\theta}-r) \stackrel{d}{\longrightarrow} \chi^{2}(v) .
$$

In Proposition 2, we already showed that $\widehat{V} \stackrel{p}{\longrightarrow} V$ and $\widehat{W} \stackrel{p}{\longrightarrow} W$. Consequently, we have

$$
T_{N, M}=N(R \widehat{\theta}-r)^{T}\left(R \widehat{W}^{-1} \widehat{V} \widehat{W}^{-1} R^{T}\right)^{-1}(R \widehat{\theta}-r) \stackrel{d}{\longrightarrow} \chi^{2}(v)
$$


Table 1. Size $\alpha$ test results for the Wald-type statistic

\begin{tabular}{llllllcccc}
\hline & & \multicolumn{4}{c}{ Under $H_{0}$} & \multicolumn{5}{c}{ Under $H_{a}$} \\
\cline { 3 - 9 }$N$ & $M$ & 0.1 & 0.05 & 0.025 & 0.01 & 0.1 & 0.05 & 0.025 & 0.01 \\
\hline 250 & 390 & 0.195 & 0.121 & 0.041 & 0.012 & 0.634 & 0.527 & 0.314 & 0.138 \\
& 2340 & 0.162 & 0.100 & 0.032 & 0.008 & 0.887 & 0.835 & 0.720 & 0.475 \\
& 4680 & 0.154 & 0.095 & 0.029 & 0.008 & 0.951 & 0.920 & 0.832 & 0.664 \\
& 23400 & 0.158 & 0.086 & 0.029 & 0.007 & 0.994 & 0.992 & 0.977 & 0.921 \\
500 & 390 & 0.146 & 0.092 & 0.035 & 0.008 & 0.880 & 0.826 & 0.628 & 0.354 \\
& 2340 & 0.115 & 0.063 & 0.016 & 0.002 & 0.991 & 0.984 & 0.963 & 0.882 \\
& 4680 & 0.128 & 0.060 & 0.017 & 0.002 & 1.000 & 0.999 & 0.988 & 0.966 \\
& 23400 & 0.132 & 0.070 & 0.019 & 0.002 & 1.000 & 1.000 & 1.000 & 0.999 \\
750 & 390 & 0.121 & 0.066 & 0.021 & 0.004 & 0.962 & 0.932 & 0.831 & 0.601 \\
& 2340 & 0.110 & 0.063 & 0.017 & 0.002 & 1.000 & 1.000 & 0.997 & 0.982 \\
& 4680 & 0.111 & 0.065 & 0.019 & 0.002 & 1.000 & 1.000 & 1.000 & 0.998 \\
& 23400 & 0.113 & 0.063 & 0.016 & 0.005 & 1.000 & 1.000 & 1.000 & 1.000 \\
& & & & & & & & & \\
1000 & 390 & 0.135 & 0.076 & 0.023 & 0.003 & 0.988 & 0.981 & 0.930 & 0.793 \\
& 2340 & 0.109 & 0.066 & 0.013 & 0.001 & 1.000 & 1.000 & 1.000 & 0.998 \\
& 4680 & 0.104 & 0.063 & 0.016 & 0.000 & 1.000 & 1.000 & 1.000 & 1.000 \\
& 23400 & 0.106 & 0.055 & 0.013 & 0.001 & 1.000 & 1.000 & 1.000 & 1.000 \\
\hline
\end{tabular}

Notes. This table presents Wald-type test rejection rates under the null and alternative hypothesis for $\alpha=0.1,0.05,0.025,0.01, N=250,500,750,1000$, and $M=390,2340,4680,23400$. 
Table 2. SG-Itô model parameter estimation and hypothesis test results based on the realized volatility estimates

\begin{tabular}{cccccccc}
\hline & \multicolumn{7}{c}{ Models } \\
\cline { 2 - 7 } Parameters & $(\mathrm{i})$ & $(\mathrm{ii})$ & $(\mathrm{iii})$ & $(\mathrm{iv})$ & $(\mathrm{v})$ & $(\mathrm{vi})$ & $(\mathrm{vii})$ \\
\hline$\omega_{1}$ & $0.024^{* * *}$ & $0.015^{* * *}$ & $0.020^{* * *}$ & $0.025^{* * *}$ & $0.024^{* * *}$ & $0.017^{* * *}$ & $0.027^{* * *}$ \\
& $(0.004)$ & $(0.005)$ & $(0.004)$ & $(0.007)$ & $(0.007)$ & $(0.006)$ & $(0.004)$ \\
$\gamma_{1}$ & $0.671^{* * *}$ & $0.679^{* * *}$ & $0.686^{* * *}$ & $0.796^{* * *}$ & $0.743^{* * *}$ & $0.734^{* * *}$ & $0.684^{* * *}$ \\
& $(0.037)$ & $(0.037)$ & $(0.034)$ & $(0.045)$ & $(0.051)$ & $(0.050)$ & $(0.038)$ \\
$\beta_{1}$ & $0.130^{* * *}$ & $0.124^{* * *}$ & $0.149^{* * *}$ & $0.154^{* * *}$ & $0.154^{* * *}$ & $0.126^{* * *}$ & $0.167^{* * *}$ \\
& $(0.024)$ & $(0.019)$ & $(0.021)$ & $(0.020)$ & $(0.026)$ & $(0.031)$ & $(0.028)$ \\
$\omega_{2}$ & $0.053^{* * *}$ & $0.039^{* * *}$ & $0.044^{* * *}$ & 0.010 & 0.010 & $0.022^{* *}$ & 0.012 \\
& $(0.014)$ & $(0.008)$ & $(0.012)$ & $(0.020)$ & $(0.019)$ & $(0.011)$ & $(0.009)$ \\
$\gamma_{2}$ & $0.814^{* * *}$ & $0.738^{* * *}$ & $0.820^{* * *}$ & $0.522^{* * *}$ & $0.702^{* * *}$ & $0.799^{* * *}$ & $0.830^{* * *}$ \\
& $(0.072)$ & $(0.048)$ & $(0.059)$ & $(0.078)$ & $(0.084)$ & $(0.071)$ & $(0.050)$ \\
$\beta_{2}$ & $0.136^{* * *}$ & $0.212^{* * *}$ & $0.130^{* * *}$ & $0.167^{* * *}$ & $0.160^{* * *}$ & $0.143^{* * *}$ & $0.120^{* * *}$ \\
& $(0.030)$ & $(0.032)$ & $(0.033)$ & $(0.042)$ & $(0.030)$ & $(0.036)$ & $(0.021)$ \\
& & & & & & & \\
Wald & $30.252^{* * *}$ & $52.567^{* * *}$ & $41.563^{* * *}$ & $32.122^{* * *}$ & 2.573 & $35.018^{* * *}$ & $10.104^{* *}$ \\
Statistic & $(0.000)$ & $(0.000)$ & $(0.000)$ & $(0.000)$ & $(0.462)$ & $(0.000)$ & $(0.018)$ \\
\hline
\end{tabular}

Notes. This table represents SG-Itô model parameter estimation and hypothesis test results based on the realized volatility estimates. Models (i)-(vii) are constructed to examine the following effects on the volatility process: (i) leverage (previous-day market return), (ii) leverage (overnight return), (iii) Chinese stock market movement, (iv) pre-holiday, (v) post-holiday, (vi) abnormal trading volume, and (vii) aggregate liquidity. The Wald-type statistics are from the Wald-type test under the null hypothesis $H_{0}:\left\{\omega_{1}=\omega_{2}, \gamma_{1}=\gamma_{2}, \beta_{1}=\beta_{2}\right\}$. For the parameter estimation, intraday S\&P 500 index data spanning from January 1, 2015, to December 31, 2018, are used. The numbers in parentheses under parameter estimates and Wald-type statistics indicate standard errors and $p$-values, respectively. ${ }^{* * *}$ and ${ }^{* *}$ on coefficients and Wald-type statistics denote statistical significance at the $1 \%$ and $5 \%$ level, respectively. 
Table 3. Integrated form of SG-Itô model parameter estimates

\begin{tabular}{cccccccc}
\hline & \multicolumn{7}{c}{ Models } \\
\cline { 2 - 8 } Parameters & (i) & (ii) & (iii) & (iv) & (v) & (vi) & (vii) \\
\hline$\omega_{11}^{h}$ & 0.026 & 0.016 & 0.022 & 0.027 & 0.026 & 0.018 & 0.029 \\
$\gamma_{11}^{h}$ & 0.671 & 0.679 & 0.686 & 0.796 & 0.743 & 0.734 & 0.684 \\
$\beta_{11}^{h}$ & 0.117 & 0.111 & 0.136 & 0.150 & 0.146 & 0.117 & 0.153 \\
& & & & & & & \\
$\omega_{12}^{h}$ & 0.039 & 0.029 & 0.033 & 0.020 & 0.019 & 0.021 & 0.020 \\
$\gamma_{12}^{h}$ & 0.749 & 0.681 & 0.769 & 0.608 & 0.716 & 0.764 & 0.785 \\
$\beta_{12}^{h}$ & 0.122 & 0.190 & 0.119 & 0.162 & 0.151 & 0.133 & 0.110 \\
& & & & & & & \\
$\omega_{21}^{h}$ & 0.042 & 0.030 & 0.035 & 0.017 & 0.019 & 0.022 & 0.022 \\
$\gamma_{21}^{h}$ & 0.729 & 0.737 & 0.732 & 0.683 & 0.728 & 0.768 & 0.723 \\
$\beta_{21}^{h}$ & 0.127 & 0.121 & 0.145 & 0.129 & 0.143 & 0.122 & 0.162 \\
& & & & & & & \\
$\omega_{22}^{h}$ & 0.057 & 0.044 & 0.046 & 0.011 & 0.011 & 0.024 & 0.012 \\
$\gamma_{22}^{h}$ & 0.814 & 0.738 & 0.820 & 0.522 & 0.702 & 0.799 & 0.830 \\
$\beta_{22}^{h}$ & 0.133 & 0.206 & 0.127 & 0.139 & 0.148 & 0.139 & 0.117 \\
\hline
\end{tabular}

Notes. This table presents the integrated form of SG-Itô model parameter estimates (i.e., $\widehat{\theta^{h}}$ ) suggested in Theorem 1(b). Models (i)-(vii) are constructed to examine the following effects on the volatility process: (i) leverage (previous-day market return), (ii) leverage (overnight return), (iii) Chinese stock market movement, (iv) pre-holiday, (v) post-holiday, (vi) abnormal trading volume, and (vii) aggregate liquidity. 
Table 4. Estimation of the GARCH, GARCH-Itô, and RS-GARCH model parameters

\begin{tabular}{cccccccccc}
\hline & & \multicolumn{8}{c}{ RS-GARCH } \\
\cline { 4 - 9 } Parameters & GARCH & GARCH-Itô & (i) & (ii) & (iii) & (iv) & (v) & (vi) & (vii) \\
\hline$\omega_{1}^{L}$ & 0.023 & 0.021 & 0.016 & 0.001 & 0.017 & 0.010 & 0.029 & 0.009 & 0.027 \\
$\gamma_{1}^{L}$ & 0.772 & 0.738 & 0.805 & 0.764 & 0.805 & 0.835 & 0.787 & 0.696 & 0.749 \\
$\beta_{1}^{L}$ & 0.204 & 0.153 & 0.043 & 0.159 & 0.070 & 0.165 & 0.178 & 0.192 & 0.212 \\
& & & & & & & & & \\
$\omega_{2}^{L}$ & & & 0.086 & 0.054 & 0.070 & 0.052 & 0.000 & 0.073 & 0.012 \\
$\gamma_{2}^{L}$ & & 0.853 & 0.800 & 0.723 & 0.613 & 0.725 & 0.767 & 0.841 \\
$\beta_{2}^{L}$ & & 0.147 & 0.200 & 0.277 & 0.330 & 0.275 & 0.233 & 0.159 \\
\hline
\end{tabular}

Notes. This table presents paremeter estimates of the GARCH, GARCH-Itô, and RS-GARCH models. The RS-GARCH models models (i)-(vii) are constructed to examine the following effects on the volatility process: (i) leverage (previous-day market return), (ii) leverage (overnight return), (iii) Chinese stock market movement, (iv) pre-holiday, (v) post-holiday, (vi) abnormal trading volume, and (vii) aggregate liquidity. 
Table 5. SG-Itô model parameter estimation and hypothesis test results based on the lowfrequency data only

\begin{tabular}{cccccccc}
\hline & \multicolumn{7}{c}{ Models } \\
\cline { 2 - 8 } Parameters & $(\mathrm{i})$ & $(\mathrm{ii})$ & $(\mathrm{iii})$ & $(\mathrm{iv})$ & $(\mathrm{v})$ & $(\mathrm{vi})$ & $(\mathrm{vii})$ \\
\hline$\omega_{1}$ & $0.018^{*}$ & 0.010 & $0.022^{*}$ & 0.031 & 0.031 & 0.010 & $0.021^{* *}$ \\
& $(0.010)$ & $(0.017)$ & $(0.012)$ & $(0.024)$ & $(0.023)$ & $(0.012)$ & $(0.010)$ \\
$\gamma_{1}$ & $0.744^{* * *}$ & $0.758^{* * *}$ & $0.705^{* * *}$ & $0.777^{* * *}$ & $0.736^{* * *}$ & $0.760^{* * *}$ & $0.707^{* * *}$ \\
& $(0.098)$ & $(0.085)$ & $(0.062)$ & $(0.067)$ & $(0.086)$ & $(0.110)$ & $(0.052)$ \\
$\beta_{1}$ & 0.123 & $0.158^{* *}$ & $0.207^{* * *}$ & $0.173^{* * *}$ & 0.214 & 0.162 & $0.243^{* * *}$ \\
& $(0.109)$ & $(0.073)$ & $(0.064)$ & $(0.045)$ & $(0.069)$ & $(0.099)$ & $(0.055)$ \\
$\omega_{2}$ & 0.115 & $0.059^{*}$ & 0.082 & 0.010 & 0.010 & 0.059 & 0.033 \\
& $(0.098)$ & $(0.035)$ & $(0.071)$ & $(0.078)$ & $(0.048)$ & $(0.072)$ & $(0.034)$ \\
$\gamma_{2}$ & $0.803^{* * *}$ & $0.704^{* * *}$ & $0.787^{* * *}$ & $0.680^{* * *}$ & $0.789^{* * *}$ & $0.710^{* * *}$ & $0.940^{* * *}$ \\
& $(0.273)$ & $(0.125)$ & $(0.148)$ & $(0.138)$ & $(0.139)$ & $(0.202)$ & $(0.093)$ \\
$\beta_{2}$ & $0.147^{* *}$ & $0.246^{* * *}$ & $0.163^{* * *}$ & $0.270^{* * *}$ & $0.161^{* *}$ & $0.240^{* *}$ & 0.010 \\
& $(0.069)$ & $(0.068)$ & $(0.046)$ & $(0.089)$ & $(0.064)$ & $(0.109)$ & $(0.048)$ \\
& & & & & & & \\
Wald & $7.139^{*}$ & 2.723 & 5.287 & 0.983 & 0.353 & $7.603^{*}$ & $9.443^{* *}$ \\
Statistic & $(0.068)$ & $(0.436)$ & $(0.152)$ & $(0.805)$ & $(0.950)$ & $(0.055)$ & $(0.024)$ \\
\hline
\end{tabular}

Notes. This table presents SG-Itô model parameter estimation and hypothesis test results based on the low-frequency data for models (i)-(vii). Models (i)-(vii) are constructed to examine the following effects on the volatility process: (i) leverage (previous-day market return), (ii) leverage (overnight return), (iii) Chinese stock market movement, (iv) pre-holiday, (v) post-holiday, (vi) abnormal trading volume, and (vii) aggregate liquidity. The Wald-type statistics are from the Wald-type test under the null hypothesis $H_{0}:\left\{\omega_{1}=\omega_{2}, \gamma_{1}=\gamma_{2}, \beta_{1}=\beta_{2}\right\}$. For the parameter estimation, daily S\&P 500 index data spanning from January 1, 2015, to December 31, 2018 are used. The numbers in parentheses under parameter estimates and Wald-type statistics indicate standard error and $p$-value, respectively. ${ }^{* * *},{ }^{* *}$, and ${ }^{*}$ on coefficients and Wald-type statistics denotes statistical significance at the $1 \%, 5 \%$, and $10 \%$ level, respectively. 
Table 6. Out-of-sample prediction performance of the volatility models measured by MAPEs

\begin{tabular}{cccccccc}
\hline & \multicolumn{6}{c}{ Out-of-sample MAPEs } \\
\cline { 2 - 8 } Volatility models & (i) & (ii) & (iii) & (iv) & (v) & (vi) & (vii) \\
\hline SG-Ito & 0.560 & 0.503 & 0.552 & 0.558 & 0.567 & 0.564 & 0.572 \\
GARCH-Ito & 0.571 & 0.571 & 0.571 & 0.571 & 0.571 & 0.571 & 0.571 \\
RS-GARCH & 0.812 & 0.766 & 0.805 & 0.930 & 0.909 & 0.917 & 0.952 \\
GARCH & 0.925 & 0.925 & 0.925 & 0.925 & 0.925 & 0.925 & 0.925 \\
HAR & 0.646 & 0.646 & 0.646 & 0.646 & 0.646 & 0.646 & 0.646 \\
\hline
\end{tabular}

Notes. This table presents out-of-sample prediction performance of the volatility models measured by MAPEs. Models (i)-(vii) are constructed to examine the following effects on the volatility process: (i) leverage (previous-day market return), (ii) leverage (overnight return), (iii) Chinese stock market movement (iv) pre-holiday, (v) post-holiday, (vi) abnormal trading volume, and (vii) aggregate liquidity. Estimation window is 750 days and prediction period is from December 22, 2017, to December 31, 2018 (248 days). 

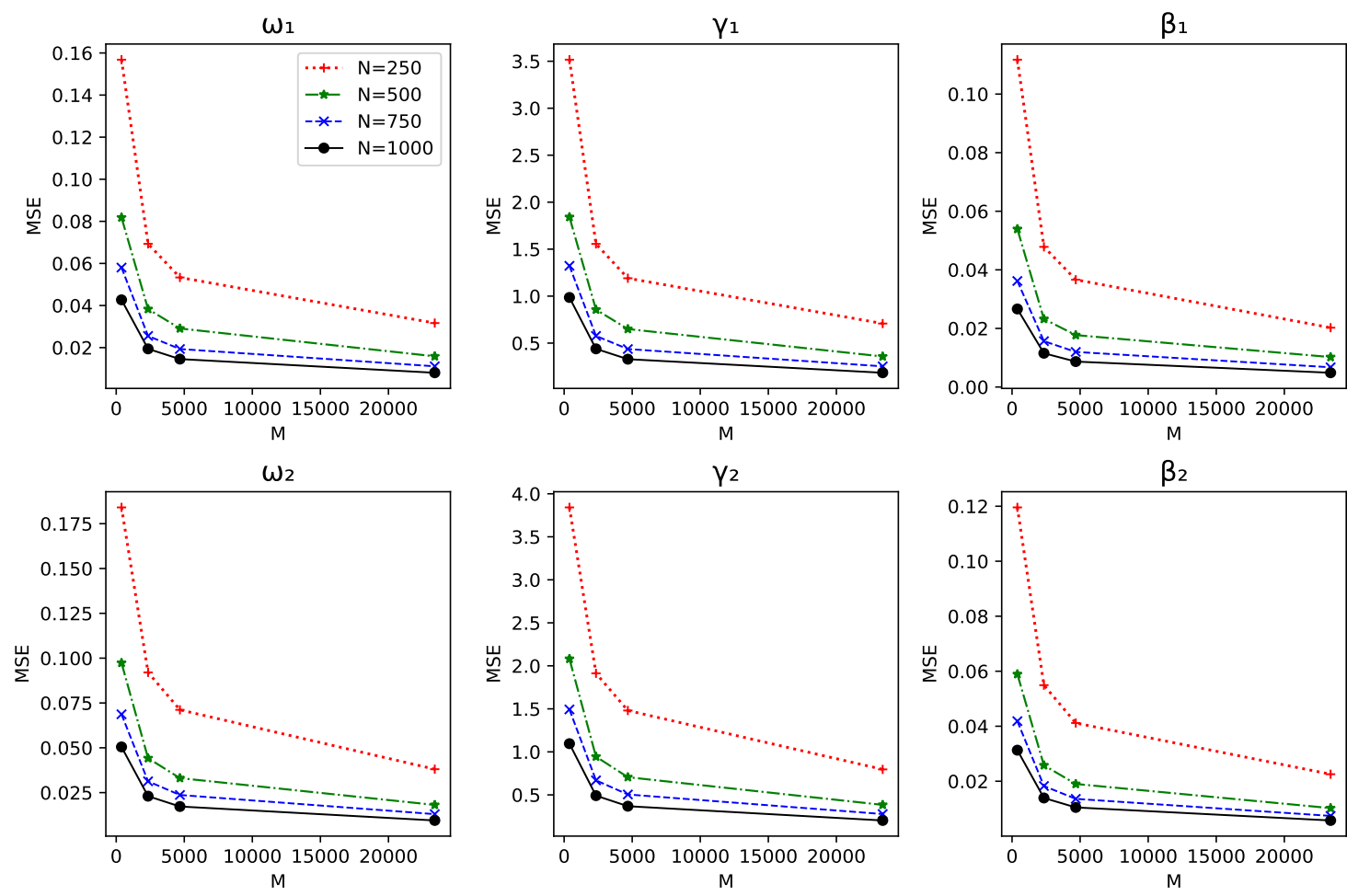

Figure 1. MSEs of parameter estimates of the SG-Itô model

Notes. This figure illustrates MSEs of parameter estimates of the SG-Itô model based on data simulated from the SG-Itô model with $N=250,500,750,1000$ and $M=390,2340,4680,23400$. 
(a) Null hypothesis

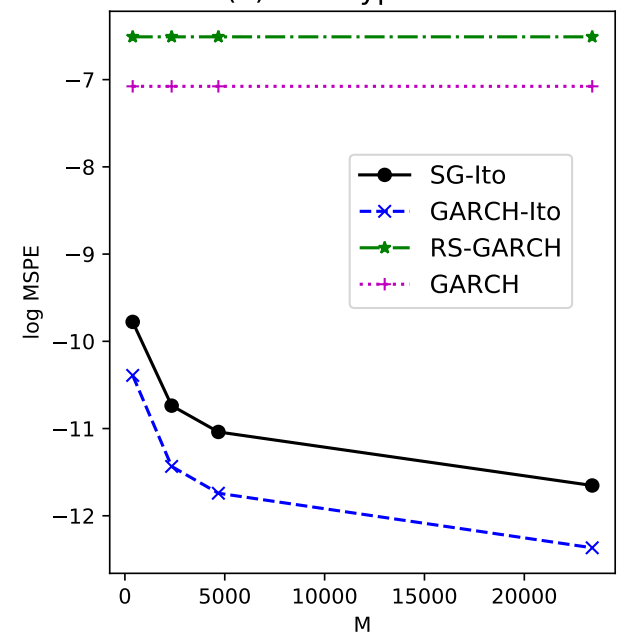

(b) Alternative hypothesis

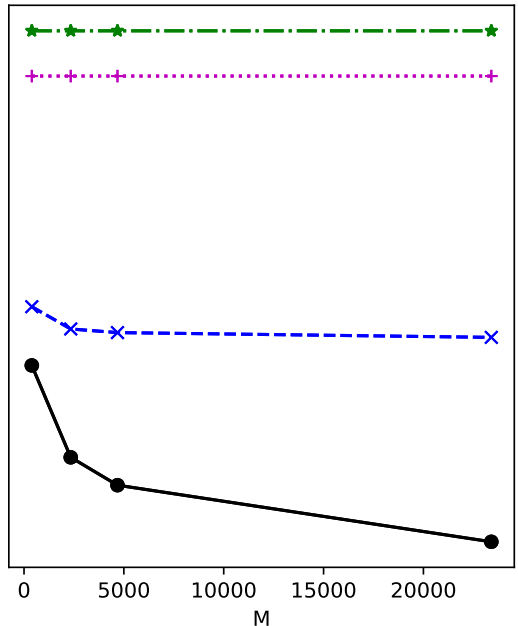

Figure 2. One-day ahead out-of-sample volatility prediction error

Notes. This figure illustrates one-day ahead out-of-sample volatility prediction error (MSPE) of volatility models against $M$ under the null and alternative hypothesis, with 500-day estimation window and prediction periods. Note that we took the log transformation of MSPEs. 

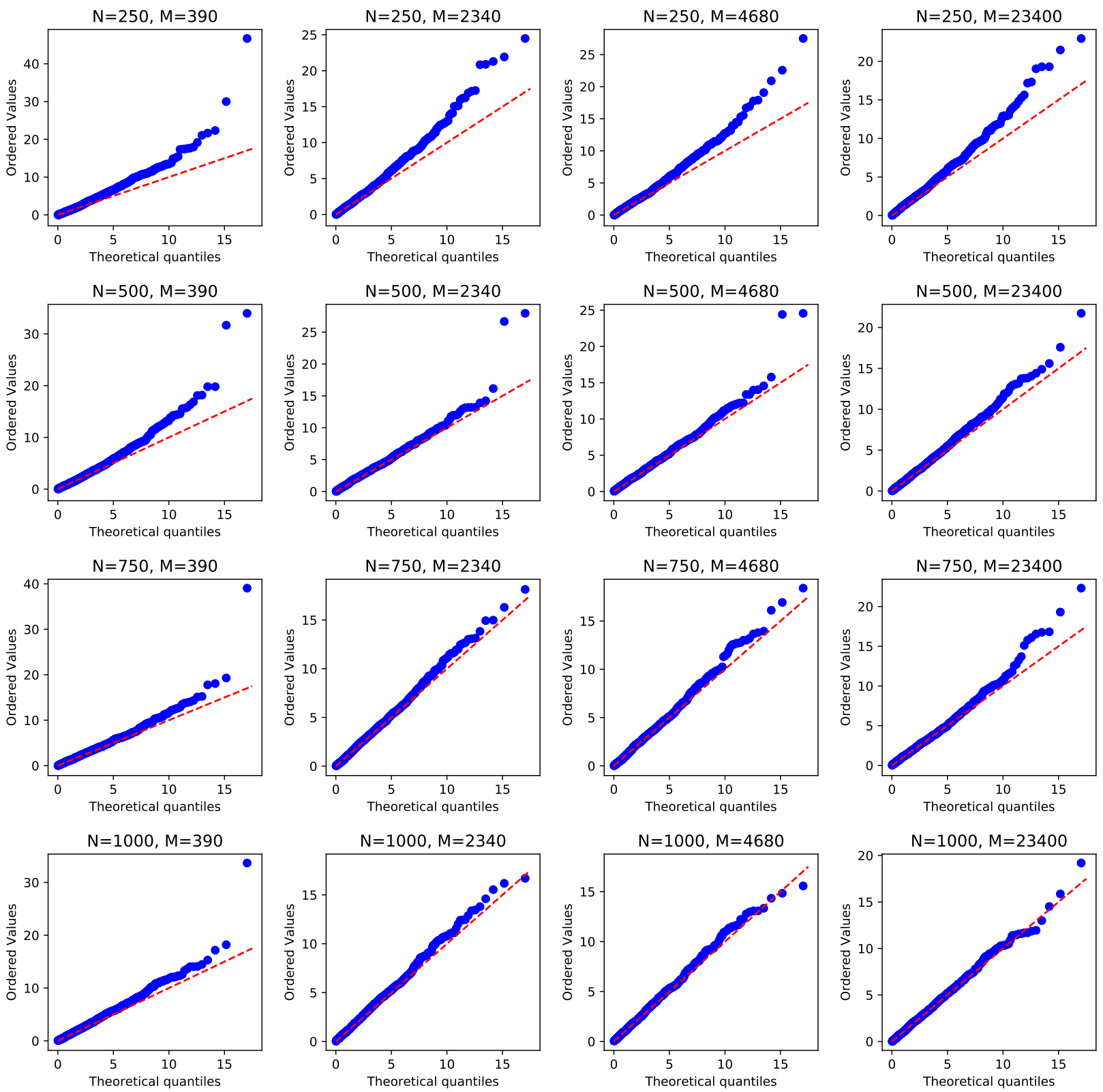

Figure 3. $\chi^{2}$ quantile-quantile plots of the Wald-type statistic

Notes. This figure illustrates $\chi^{2}$ quantile-quantile plots of the Wald-type statistic under the null hypothesis for $N=250,500,750,1000$, and $M=390,2340,4680,23400$. The real line denotes the best linear fitted line which illustrates perfect $\chi^{2}$ distribution. 


\section{Data availability statement}

The S\&P500 intraday index data is provided by the Chicago Board of Exchange (CBOE).

(web link: https://datashop.cboe.com/). Please note that the data sharing policy of CBOE restricts the redistribution of data.

\section{References}

Abraham, A. and Ikenberry, D. L. (1994). The individual investor and the weekend effect. Journal of Financial and Quantitative Analysis, 29(2):263-277.

Ahoniemi, K., Fuertes, A.-M., and Olmo, J. (2016). Overnight news and daily equity trading risk limits. Journal of Financial Econometrics, 14(3):525-551.

Ahoniemi, K. and Lanne, M. (2013). Overnight stock returns and realized volatility. International Journal of Forecasting, 29(4):592-604.

Aït-Sahalia, Y., Fan, J., and Xiu, D. (2010). High-frequency covariance estimates with noisy and asynchronous financial data. Journal of the American Statistical Association, 105(492):15041517.

Andersen, T. G. and Bollerslev, T. (1998). Answering the skeptics: Yes, standard volatility models do provide accurate forecasts. International Economic Review, pages 885-905.

Andrei, D. and Hasler, M. (2014). Investor attention and stock market volatility. Review of Financial Studies, 28(1):33-72.

Andrews, D. W. (1992). Generic uniform convergence. Econometric Theory, 8(2):241-257.

Barber, B. M. and Odean, T. (2007). All that glitters: The effect of attention and news on the buying behavior of individual and institutional investors. Review of Financial Studies, 21(2):785818. 
Barndorff-Nielsen, O. E., Hansen, P. R., Lunde, A., and Shephard, N. (2008). Designing realized kernels to measure the ex post variation of equity prices in the presence of noise. Econometrica, 76(6):1481-1536.

Bauwens, L., Dufays, A., and Rombouts, J. V. (2014). Marginal likelihood for markov-switching and change-point garch models. Journal of Econometrics, 178:508-522.

Bauwens, L., Preminger, A., and Rombouts, J. V. (2010). Theory and inference for a markov switching garch model. Econometrics Journal, 13(2):218-244.

Berument, H. and Kiymaz, H. (2001). The day of the week effect on stock market volatility. Journal of Economics and Finance, 25(2):181-193.

Birru, J. (2018). Day of the week and the cross-section of returns. Journal of Financial Economics, 130(1):182-214.

Black, F. (1976). Studies of stock market volatility changes. 1976 Proceedings of the American Statistical Association Bisiness and Economic Statistics Section.

Bollerslev, T. (1986). Generalized autoregressive conditional heteroskedasticity. Journal of Econometrics, 31(3):307-327.

Braun, P. A., Nelson, D. B., and Sunier, A. M. (1995). Good news, bad news, volatility, and betas. Journal of Finance, 50(5):1575-1603.

Carr, P. and Wu, L. (2017). Leverage effect, volatility feedback, and self-exciting market disruptions. Journal of Financial and Quantitative Analysis, 52(5):2119-2156.

Cerovecki, C., Francq, C., Hörmann, S., and Zakoian, J.-M. (2019). Functional garch models: The quasi-likelihood approach and its applications. Journal of Econometrics, 209(2):353-375.

Chen, Y., Eaton, G. W., and Paye, B. S. (2018). Micro (structure) before macro? the predictive power of aggregate illiquidity for stock returns and economic activity. Journal of Financial Economics, 130(1):48-73. 
Christensen, K., Kinnebrock, S., and Podolskij, M. (2010). Pre-averaging estimators of the ex-post covariance matrix in noisy diffusion models with non-synchronous data. Journal of Econometrics, 159(1):116-133.

Christie, A. A. (1982). The stochastic behavior of common stock variances: Value, leverage and interest rate effects. Journal of Financial Economics, 10(4):407-432.

Copeland, T. E. (1976). A model of asset trading under the assumption of sequential information arrival. Journal of Finance, 31(4):1149-1168.

Corsi, F. (2009). A simple approximate long-memory model of realized volatility. Journal of Financial Econometrics, 7(2):174-196.

Corwin, S. A. and Schultz, P. (2012). A simple way to estimate bid-ask spreads from daily high and low prices. Journal of Finance, 67(2):719-760.

Engle, R. F. (1982). Autoregressive conditional heteroscedasticity with estimates of the variance of united kingdom inflation. Econometrica, pages 987-1007.

Engle, R. F. and Gallo, G. M. (2006). A multiple indicators model for volatility using intra-daily data. Journal of Econometrics, 131(1-2):3-27.

Fan, J. and Kim, D. (2018). Robust high-dimensional volatility matrix estimation for high-frequency factor model. Journal of the American Statistical Association, 113(523):1268-1283.

Figlewski, S. and Wang, X. (2000). Is the 'leverage effect' a leverage effect? Available at SSRN 256109.

French, K. R. (1980). Stock returns and the weekend effect. Journal of Financial Economics, 8(1):55-69.

Gallant, A. R., Rossi, P. E., and Tauchen, G. (1992). Stock prices and volume. Review of Financial Studies, 5(2):199-242. 
Glosten, L. R., Jagannathan, R., and Runkle, D. E. (1993). On the relation between the expected value and the volatility of the nominal excess return on stocks. Journal of Finance, 48(5):17791801.

Gray, S. F. (1996). Modeling the conditional distribution of interest rates as a regime-switching process. Journal of Financial Economics, 42(1):27-62.

Haas, M., Mittnik, S., and Paolella, M. S. (2004). A new approach to markov-switching garch models. Journal of Financial Econometrics, 2(4):493-530.

Hall, P. and Heyde, C. C. (2014). Martingale limit theory and its application. Academic press.

Hamilton, J. D. and Susmel, R. (1994). Autoregressive conditional heteroskedasticity and changes in regime. Journal of Econometrics, 64(1-2):307-333.

Hansen, P. R., Huang, Z., and Shek, H. H. (2012). Realized garch: a joint model for returns and realized measures of volatility. Journal of Applied Econometrics, 27(6):877-906.

Jacod, J., Li, Y., Mykland, P. A., Podolskij, M., and Vetter, M. (2009). Microstructure noise in the continuous case: The pre-averaging approach. Stochastic Processes and Their Applications, 119(7):2249-2276.

Jennings, R. H., Starks, L. T., and Fellingham, J. C. (1981). An equilibrium model of asset trading with sequential information arrival. Journal of Finance, 36(1):143-161.

Kallsen, J. and Taqqu, M. S. (1998). Option pricing in arch-type models. Mathematical Finance, $8(1): 13-26$.

Kambouroudis, D. S. and McMillan, D. G. (2016). Does vix or volume improve garch volatility forecasts? Applied Economics, 48(13):1210-1228.

Karpoff, J. M. (1987). The relation between price changes and trading volume: A survey. Journal of Financial and Quantitative Analysis, 22(1):109-126. 
Kim, D. (2016). Statistical inference for unified garch-itô models with high-frequency financial data. Journal of Time Series Analysis, 37(4):513-532.

Kim, D. and Fan, J. (2019). Factor garch-itô models for high-frequency data with application to large volatility matrix prediction. Journal of econometrics, 208(2):395-417.

Kim, D. and Kon, S. J. (1994). Alternative models for the conditional heteroscedasticity of stock returns. Journal of Business, pages 563-598.

Kim, D., Liu, Y., and Wang, Y. (2018). Large volatility matrix estimation with factor-based diffusion model for high-frequency financial data. Bernoulli, 24(4B):3657-3682.

Kim, D. and Wang, Y. (2016). Unified discrete-time and continuous-time models and statistical inferences for merged low-frequency and high-frequency financial data. Journal of Econometrics, 194(2):220-230.

Kim, D. and Wang, Y. (2020). Overnight volatility processes. Manuscript.

Kim, D., Wang, Y., and Zou, J. (2016). Asymptotic theory for large volatility matrix estimation based on high-frequency financial data. Stochastic Processes and their Applications, 126(11):3527-3577.

Kiymaz, H. and Berument, H. (2003). The day of the week effect on stock market volatility and volume: International evidence. Review of Financial Economics, 12(4):363-380.

Klaassen, F. (2002). Improving garch volatility forecasts with regime-switching garch. Empirical Economics, 27:363-394.

Lai, Y.-S., Sheu, H.-J., and Lee, H.-T. (2017). A multivariate markov regime-switching highfrequency-based volatility model for optimal futures hedging. Journal of Futures Markets, 37(11):1124-1140.

Lakonishok, J. and Maberly, E. (1990). The weekend effect: Trading patterns of individual and institutional investors. The Journal of Finance, 45(1):231-243. 
Lamoureux, C. G. and Lastrapes, W. D. (1990a). Heteroskedasticity in stock return data: Volume versus garch effects. Journal of Finance, 45(1):221-229.

Lamoureux, C. G. and Lastrapes, W. D. (1990b). Persistence in variance, structural change, and the garch model. Journal of Business \& Economic Statistics, 8(2):225-234.

Lamoureux, C. G. and Lastrapes, W. D. (1994). Endogenous trading volume and momentum in stock-return volatility. Journal of Business \& Economic Statistics, 12(2):253-260.

Lange, T. and Rahbek, A. (2009). An introduction to regime switching time series models. In Handbook of Financial Time Series, pages 871-887. Springer.

Lee, S.-W. and Hansen, B. E. (1994). Asymptotic theory for the garch (1, 1) quasi-maximum likelihood estimator. Econometric Theory, 10(1):29-52.

Liu, Z., Kong, X.-B., and Jing, B.-Y. (2018). Estimating the integrated volatility using highfrequency data with zero durations. Journal of Econometrics, 204(1):18-32.

Merlevede, F. and Peligrad, M. (2000). The functional central limit theorem under the strong mixing condition. Annals of Probability, pages 1336-1352.

Miller, E. M. (1988). Why a weekend effect? Journal of Portfolio Management, 14(4):43.

Nelson, D. B. (1990). Arch models as diffusion approximations. Journal of Econometrics, 45(12):7-38.

Nyberg, H. (2012). Risk-return tradeoff in us stock returns over the business cycle. Journal of Financial and Quantitative Analysis, 47(1):137-158.

Renault, E. and Werker, B. J. (2011). Causality effects in return volatility measures with random times. Journal of Econometrics, 160(1):272-279.

Shephard, N. and Sheppard, K. (2010). Realising the future: Forecasting with high-frequency-based volatility (heavy) models. Journal of Applied Econometrics, 25(2):197-231. 
Shi, Y. and Ho, K.-Y. (2015). Modeling high-frequency volatility with three-state figarch models. Economic Modelling, 51:473-483.

Song, X., Kim, D., Yuan, H., Cui, X., Lu, Z., Zhou, Y., and Wang, Y. (2021). Volatility analysis with realized garch-itô models. To be appeared in Journal of Econometrics.

Tao, M., Wang, Y., and Chen, X. (2013). Fast convergence rates in estimating large volatility matrices using high-frequency financial data. Econometric Theory, 29(4):838-856.

Tao, M., Wang, Y., Yao, Q., and Zou, J. (2011). Large volatility matrix inference via combining low-frequency and high-frequency approaches. Journal of the American Statistical Association, 106(495):1025-1040.

Tauchen, G., Zhang, H., and Liu, M. (1996). Volume, volatility, and leverage: A dynamic analysis. Journal of Econometrics, 74(1):177-208.

Taylor, N. (2007). A note on the importance of overnight information in risk management models. Journal of Banking \& Finance, 31(1):161-180.

Tsiakas, I. (2008). Overnight information and stochastic volatility: A study of european and us stock exchanges. Journal of Banking \& Finance, 32(2):251-268.

Visser, M. P. (2011). Garch parameter estimation using high-frequency data. Journal of Financial Econometrics, 9(1):162-197.

Wang, G. H. and Yau, J. (2000). Trading volume, bid-ask spread, and price volatility in futures markets. Journal of Futures Markets, 20(10):943-970.

Wang, Y. (2002). Asymptotic nonequivalence of garch models and diffusions. Annals of Statistics, $30(3): 754-783$.

Xiu, D. (2010). Quasi-maximum likelihood estimation of volatility with high frequency data. Journal of Econometrics, 159(1):235-250. 
Zhang, L. (2006). Efficient estimation of stochastic volatility using noisy observations: A multi-scale approach. Bernoulli, 12(6):1019-1043.

Zhang, L. (2011). Estimating covariation: Epps effect, microstructure noise. Journal of Econometrics, 160(1):33-47.

Zhang, X. and Frey, R. (2015). Improving armagarch forecasts for high frequency data with regimeswitching arma-garch. Journal of Computational Analysis $\& 3$ Applications, 18(1):p727. 